TRANSACTIONS OF THE

AMERICAN MATHEMATICAL SOCIETY

Volume 356, Number 5 , Pages 1909-1935

S 0002-9947(03)03349-X

Article electronically published on July 24, 2003

\title{
AUTOMORPHIC FORMS AND DIFFERENTIABILITY PROPERTIES
}

\author{
FERNANDO CHAMIZO
}

\begin{abstract}
We consider Fourier series given by a type of fractional integral of automorphic forms, and we study their local and global properties, especially differentiability and fractal dimension of the graph of their real and imaginary parts. In this way we can construct fractal objects and continuous non-differentiable functions associated with elliptic curves and theta functions.
\end{abstract}

\section{§1. INTRODUCTION}

"Riemann's example", the real function defined as

$$
F(x)=\sum_{n=1}^{\infty} \frac{\sin \left(2 \pi n^{2} x\right)}{n^{2}},
$$

is a trigonometric series whose history that can be traced back to the origins of the theory of functions in the 19th century. Apparently $F$ was initially introduced as an example of a continuous but nowhere differentiable function, and it has motivated a vast literature (see the references in 2]) culminating in several works proving that $F$ is a fractal-like continuous function which is differentiable only at rational values of the form $a /(2 b)$ with $a$ and $b$ odd integers. According to Weierstrass [16, this latter fact contradicted a claim of Riemann (but the extensive historical analysis in [2] does not support the claim of Weierstrass).

In this paper we shall present general results that allow us to determine the differentiability and other local and global properties of a wide class of functions. In this setting Riemann's example will be only a simple particular case (see the last comments in $\S 2$ ).

In order to illustrate the diversity of the functions considered, we mention the following examples:

$$
F_{1}(x)=\sum_{n \equiv \pm 1(12)} \frac{\sin \left(2 \pi n^{2} x\right)}{n^{2}}-\sum_{n \equiv \pm 5(12)} \frac{\sin \left(2 \pi n^{2} x\right)}{n^{2}},
$$

where $n \equiv a(b)$ means " $b$ divides $n-a$ ".

$$
F_{2}(x)=\sum_{n=1}^{\infty} \frac{a_{n}}{n^{8 / 5}} \sin (2 \pi n x),
$$

Received by the editors May 14, 2002 and, in revised form, March 27, 2003.

2000 Mathematics Subject Classification. Primary 42A16, 11F12, 28A80.

(C)2003 American Mathematical Society 
where $a_{n}$ are the coefficients of the Hasse-Weil $L$-function of the elliptic curve $E: y^{2}=x^{3}+1$.

$$
F_{3}(x)=\sum_{n=1}^{\infty} \frac{c_{n}}{n^{20 / 3}} \cos (2 \pi n x),
$$

where $c_{n}$ is the $n$-th coefficient in the power series expansion of $x \prod_{k=1}^{\infty}\left(1-x^{k}\right)^{24}$.

$$
F_{4}(x)=\sum_{n=1}^{\infty} \frac{r(n)}{n^{5 / 4}} e^{2 \pi i n x},
$$

where $r(n)=\left|\left\{(x, y) \in \mathbf{Z}^{2}: n=x^{2}+2 y^{2}\right\}\right|$.

A direct consequence of our main results is that $F_{1}, F_{2}$ and $F_{3}$ are continuous functions which are differentiable in $\mathbf{Q}$ and non-differentiable on $\mathbf{R}-\mathbf{Q}$; moreover the graphs of these functions are fractals with dimensions $5 / 4,7 / 5$ and $4 / 3$, respectively.

Finer properties can be specified in each case, for instance:

a) $F_{1}$ is not only a simple example of a continuous function having $\mathbf{Q}$ as its set of differentiability (such examples usually do not appear in calculus textbooks) but also its derivative vanishes on $\mathbf{Q}$.

b) The value of the derivative of $F_{2}$ on $\mathbf{Q}$ is related to special values of the HasseWeil $L$-function of $E$ and its twists. To illustrate this fact in an arithmetic way, we mention that if $8 / 5$ is replaced by 2 in the definition of $F_{2}$, and $E$ by an arbitrary elliptic curve over $\mathbf{Q}$, then $E$ contains finitely many rational points whenever the resulting function has a non-vanishing derivative at $x=0$.

c) The remarkable fact about $F_{3}$ is that the size of the coefficients $c_{n}$ remained as a conjecture from the time of Ramanujan to the celebrated work of P. Deligne on the Riemann hypothesis over finite fields. Nevertheless with comparatively simple arguments, some analytic properties of $F_{3}$ can be proved. For instance, $F_{3} \in \Lambda_{\beta}$ for $\beta<2 / 3$ and $F_{3}(a / b+h)-F_{3}(a / b)=c h+O\left(|h|^{4 / 3}\right)$, for $a / b \in \mathbf{Q}$.

d) Finally, $F_{4}$ has a more complicated differentiability set. Namely, it is not differentiable on $\mathbf{R}-\mathbf{Q}$ and it is differentiable at $x_{0}=a / b$ (a reduced fraction) if and only if the remainder when $b$ is divided by 8 is even and non-zero.

The common aspect in these apparently unrelated examples is that each is a kind of fractional integral of the Fourier expansion (at $i \infty$ ) of some automorphic form. Namely, $F_{1}$ corresponds to the $\eta$-function, $F_{2}$ to the newform associated to the modular curve $E: y^{2}=x^{3}+1, F_{3}$ to the discriminant function $\left(c_{n}\right.$ is the Ramanujan $\tau$-function) and $F_{4}$ to a 2 -dimensional $\theta$-function. In this paper we shall use number theoretical tools (especially the theory of automorphic forms) to determine, in general, differentiability, Lipschitz and fractal properties in this setting.

The contents of the subsequent sections are as follows: In $\S 2$ we introduce some basic notation and we state our main results, which are proved in $\S 4$ (after introducing some auxiliary results in $\S 3$ ). We devote $\S 5$ to a closer study of the Fourier series arising from theta functions. Finally, $\S 6$ can be considered as an appendix including some computer graphics with comments.

\section{§2. Notation AND Main Results}

We shall denote by $\mathcal{M}_{r}(\Gamma, m)$ the space of automorphic forms of weight $r>0$ (not necessarily integral) with respect to the Fuchsian group $\Gamma$ and having multiplier 
system $m$ (see [10]), i.e., if $f \in \mathcal{M}_{r}(\Gamma, m)$, we have

$$
f(\gamma z)=m(\gamma)\left(j_{\gamma}(z)\right)^{r} f(z) \quad \text { for every } \gamma=\left(\begin{array}{ll}
a & b \\
c & d
\end{array}\right) \in \Gamma .
$$

At each cusp $\mathfrak{a}$ of $\Gamma, f$ admits an expansion (see [10])

$$
f\left(\sigma_{\mathfrak{a}} z\right)=e\left(\kappa_{\mathfrak{a}} z\right)\left(j_{\sigma_{\mathfrak{a}}}(z)\right)^{r} \sum_{n=0}^{\infty} a_{n}^{\mathfrak{a}} e(n z),
$$

where $\sigma_{\mathfrak{a}}$ is the scaling matrix and $e(t)$ is an abbreviation for $e^{2 \pi i t}$. The constants $0 \leq \kappa_{\mathfrak{a}}<1$ and $a_{n}^{\mathfrak{a}}$ depend only on the equivalence class of $\mathfrak{a}$ under $\Gamma$.

We shall say that $f \in \mathcal{M}_{r}(\Gamma, m)$ is cuspidal at $x \in \mathbf{R} \cup\{i \infty\}$ if $\mathfrak{a}=x$ is a cusp for $\Gamma$ and $a_{0}^{\mathfrak{a}}=0$ whenever $\kappa_{\mathfrak{a}}=0$. If $f$ is cuspidal at every cusp, then $f$ is said to be a cusp form, and we shall write $f \in \mathcal{S}_{r}(\Gamma, m)$. Usually, we shall abbreviate $\mathcal{M}_{r}(\Gamma, m)$ and $\mathcal{S}_{r}(\Gamma, m)$ by $\mathcal{M}_{r}$ and $\mathcal{S}_{r}$.

In this paper we shall consider $\Gamma$ to be a subgroup of $S L_{2}(\mathbf{Z})$ of finite index containing the unit translation $\gamma(z)=z+1$ with $m(\gamma)=1$. Under these conditions the cusps for $\Gamma$ are $\mathbf{Q} \cup\{i \infty\}$, and any $f \in \mathcal{M}_{r}$ admits a Fourier expansion

$$
f(z)=\sum_{n=0}^{\infty} a_{n} e(n z) .
$$

For $f \in \mathcal{M}_{r}-\{0\}$ and $x \in \mathbf{R}$ we consider the following scaled $\alpha$-fractional integral of $f$, when it converges:

$$
f_{\alpha}(x)=\sum_{n=1}^{\infty} \frac{a_{n}}{n^{\alpha}} e(n x) .
$$

It turns out that $f_{\alpha}$ is a well defined continuous function if and only if $f \in \mathcal{S}_{r}$ with $\alpha>r / 2$ or $f \in \mathcal{M}_{r}-\mathcal{S}_{r}$ with $\alpha>r$ (see Proposition 3.1). Consequently we shall refer to these conditions as convergence conditions.

Throughout this paper, the letters $C$ and $K$ with different subscripts and superscripts will be used to denote constants (positive if the contrary is not explictly indicated, and not necessarily the same on each appearance).

The symbols $W^{n, p}=W^{n, p}([0,1]), \Lambda_{\beta}=\Lambda_{\beta}([0,1]), \lambda_{\beta}=\lambda_{\beta}([0,1])$ will denote the corresponding Sobolev space (see [1]) and Lipschitz spaces (see II.3 in [17]). The localization of these latter in $x_{0}$ will be represented by $\Lambda_{\beta}\left(x_{0}\right)$ and $\lambda_{\beta}\left(x_{0}\right)$, i.e., in Landau notation, which we shall use extensively,

$$
\begin{aligned}
& g \in \Lambda_{\beta}\left(x_{0}\right) \Leftrightarrow g(x)-g\left(x_{0}\right)=O\left(\left|x-x_{0}\right|^{\beta}\right), \\
& g \in \lambda_{\beta}\left(x_{0}\right) \Leftrightarrow g(x)-g\left(x_{0}\right)=o\left(\left|x-x_{0}\right|^{\beta}\right)
\end{aligned}
$$

as $x \rightarrow x_{0}$. In the same way, $\Lambda_{\beta}^{\log }$ will indicate the space of functions such that $g(x)-g(y)=O\left(|x-y|^{\beta}|\log | x-y||\right)$ as $x \rightarrow y$, uniformly on $y$, and $\Lambda_{\beta}^{\log }\left(x_{0}\right)$ will represent its localization at $x_{0}$.

Finally we shall introduce some concepts related to fractal dimension.

Given a continuous function $g:[0,1] \rightarrow \mathbf{R}$, we define its $h$-variation, $0<h<1$, in an interval $I \subset[0,1]$ as

$$
V(g, h ; I)=\sup _{x_{j}, y_{j}} \sum_{j}\left|g\left(x_{j}\right)-g\left(y_{j}\right)\right|,
$$

where $x_{j}, y_{j} \in[(j-1) h, j h] \cap I, j=1,2,3, \ldots$. 
We shall abbreviate $V(g, h ;[0,1])$ by $V(g, h)$. Note that the number of cells of the lattice $h \mathbf{Z} \times h \mathbf{Z}$ needed to cover the graph, $\mathcal{G}$, of $g$ differs from $h^{-1} V(g, h)$ by $O\left(h^{-1}\right)$, and hence the fractal (Minkowski, box counting) dimension of $\mathcal{G}$ is given by

$$
\operatorname{dim}(\mathcal{G})=\lim _{h \rightarrow 0^{+}} \frac{\log \left(h^{-1}+h^{-1} V(g, h)\right)}{-\log h} .
$$

Even if this limit does not exist, we can always define upper and lower dimensions, $\overline{\operatorname{dim}}(\mathcal{G})$ and $\underline{\operatorname{dim}}(\mathcal{G})$, by changing $\lim$ to $\varlimsup$ lim and $\underline{\lim }$.

Now we state our main results. As before, $f$ will represent an element of $\mathcal{M}_{r}-\{0\}$ and it will be implicitly assumed that convergence conditions are fulfilled.

Theorem 2.1. If $f_{\alpha} \in \lambda_{\beta}\left(x_{0}\right)$ with $\beta=\alpha-r / 2<1$, then $f$ is cuspidal at $x_{0}$. Moreover, if $f$ is a cusp form, $f_{\alpha} \in \Lambda_{\beta}^{\log }-\lambda_{\beta}$.

Corollary 2.1.1. For $\alpha<r / 2+1, f_{\alpha}$ is not differentiable at irrationals.

Remark. This result cannot be extended to $\alpha>r / 2+1$ because Lemma $3.2 \mathrm{c}$ ), d) implies that, in this range, $f_{\alpha} \in W^{1,2}$ for $f \in \mathcal{M}_{r}$ with $r<1$ or $f \in \mathcal{S}_{r}$.

Theorem 2.2. For $(r+1) / 2<\alpha<r / 2+1, f_{\alpha}$ is differentiable at $x_{0}$ if and only if $f$ is cuspidal at $x_{0}$. In this case the value of the derivative is

$$
f_{\alpha}^{\prime}\left(x_{0}\right)=\frac{(2 \pi)^{\alpha}}{\Gamma(\alpha)} \int_{0}^{\infty} t^{\alpha-1} f^{\prime}\left(x_{0}+i t\right) d t
$$

Remark. In fact we shall prove the stronger result that

$$
f_{\alpha}\left(x_{0}+h\right)-f_{\alpha}\left(x_{0}\right)=h \frac{(2 \pi)^{\alpha}}{\Gamma(\alpha)} \int_{0}^{\infty} t^{\alpha-1} f^{\prime}\left(x_{0}+i t\right) d t+O\left(h^{2 \alpha-r}+h^{2}\right)
$$

when $f$ is cuspidal at $x_{0}$, which suggests that the range $(r+1) / 2<\alpha<r / 2+1$ is the natural one on which to study differentiability (see the remark after Corollary 2.1.1 and note that, in fact, by Lemma $3.2 \mathrm{a}), f_{\alpha}$ is everywhere differentiable for $\alpha>r / 2+1$ if $\left.f \in S_{r}\right)$.

Corollary 2.2.1. For $(r+1) / 2<\alpha<r / 2+1$ and $f \in S_{r}, f_{\alpha}$ is differentiable in $\mathbf{Q}$ and non-differentiable in $\mathbf{R}-\mathbf{Q}$.

For $f=\sum a_{n} e(n z) \in S_{r}$, the $L$-function $L(f, s)$ is defined to be the entire continuation of $\sum a_{n} n^{-s}$ (see [1]). Given a primitive character, $\chi$, for some groups and multiplier systems appearing in the classical setting (see $\S 7.4[10]), f=$ $\sum a_{n} e(n z) \in S_{r}(\Gamma, m)$ implies that the twisted series $\sum a_{n} \bar{\chi}(n) e(n z) \in S_{r}\left(\Gamma^{\prime}, m^{\prime}\right)$. If this is the case (as we assume in the second part of the next result), we denote the latter automorphic form by $f_{\bar{\chi}}$.

Corollary 2.2.2. For $f \in S_{r}$ and $\alpha>(r+1) / 2$, the value of the derivative of $f_{\alpha}$ at the rationals satisfies

$$
f_{\alpha}^{\prime}(0)=2 \pi i L(f, \alpha-1)
$$

and

$$
\sum_{a=1}^{b} \chi(a) f_{\alpha}^{\prime}\left(\frac{a}{b}\right)=2 \pi i \tau(\chi) L\left(f_{\bar{\chi}}, \alpha-1\right),
$$

where $\chi$ is a primitive character modulo $b$ and $\tau(\chi)$ is its Gauss sum. 
Corollary 2.2.3. If $r<1$, the value of $f_{1}^{\prime}(x)$ is $-2 \pi i a_{0}$ at every point where it exists.

In the next two results we shall assume that $f$ has real Fourier coefficients. In this case $\Re f_{\alpha}$ and $\Im f_{\alpha}$ are cosine and sine real Fourier series respectively. Let $g_{\alpha}: \mathbf{R} \rightarrow \mathbf{R}$ be either of them.

Corollary 2.2.4. For $(r+1) / 2<\alpha<r / 2+1, g_{\alpha}$ is differentiable at $x_{0}$ if and only if $f$ is cuspidal at $x_{0}$.

Now we shall establish that the graph of $g_{\alpha}$ is a fractal set. The non-cuspidal case appears to be more difficult to treat, and we shall only cover the range $r \leq 1$.

Theorem 2.3. Let $\mathcal{G}$ be the graph of $g_{\alpha}$. For $f \in \mathcal{M}_{r}-\mathcal{S}_{r}$ with $r \leq 1$ or $f \in \mathcal{S}_{r}$ (without restrictions on $r$ )

$$
\operatorname{dim}(\mathcal{G})=\max (1,2-\alpha+r / 2) .
$$

The relevance of automorphic forms in modern number theory suggests several applications and reformulations of the previous theorems. We mention here the case of elliptic curves.

Eichler-Shimura theory assigns an automorphic form $f \in S_{2}\left(\Gamma_{0}(N)\right)$ to any abelian variety over $\mathbf{Q}$ appearing as a factor of the Jacobian of $X_{0}(N)$ (see $\S 7.5$ of [14), in such a way that the coefficients of its Hasse-Weil $L$-function coincide with the (normalized) Fourier coefficients of $f$. If one of these abelian varieties is one-dimensional, then it is an elliptic curve and it is said to be modular. One of the major achievements of contemporary mathematics is the recently proved ShimuraTaniyama-Weil conjecture establishing that, in fact, every elliptic curve over $\mathbf{Q}$ is modular. The $L$-function $L(f, s)$ corresponding to the automorphic form associated to an elliptic curve $E / \mathbf{Q}$, which coincides with its Hasse-Weil $L$-function, reflects some of its arithmetical properties. For instance, after the work of Kolyvagin, $L(f, 1) \neq 0$ implies that $E$ has vanishing rank, i.e., $E$ contains finitely many rational points.

After these comments, the previous results at once imply

Proposition 2.4. Let $E / \mathbf{Q}$ be a modular elliptic curve and $\sum a_{n} n^{-s}$ its HasseWeil L-function. Consider the real functions

$$
A_{\alpha}(x)=\sum \frac{a_{n}}{n^{\alpha}} \cos (2 \pi n x), \quad B_{\alpha}(x)=\sum \frac{a_{n}}{n^{\alpha}} \sin (2 \pi n x) .
$$

Then the following results hold: i) $A_{\alpha}$ and $B_{\alpha}$ are differentiable in $\mathbf{Q}$ and nondifferentiable in $\mathbf{R}-\mathbf{Q}$ for $3 / 2<\alpha<2$; ii) if $B_{2}^{\prime}(0) \neq 0$ then $E$ has vanishing rank; and iii) for $1<\alpha<2$, the graphs of $A_{\alpha}$ and $B_{\alpha}$ are fractal sets of dimension $3-\alpha$.

In principle, the slow convergence of the arising series suggests that pictures of these fractal graphs might be difficult to approximate with a personal computer; but using the PARI package they can be drawn in a reasonable amount of time. In $\S 6$ we shall check that for elliptic curves of low conductor it is possible to observe in these pictures an $x^{1+\delta} \sin \left(x^{-1}\right)$-like oscillation (the so-called "trigonometric chirps") at differentiability points with small denominator. 
To finish this section we illustrate our results, applying them to "Riemann's example"

$$
F(x)=\sum_{n=1}^{\infty} \frac{\sin \left(2 \pi n^{2} x\right)}{n^{2}}
$$

obtaining results that had been established earlier by several authors.

Note that $F(x)=\frac{1}{2} \Im \theta_{1}(x)$, where $\theta(z)=\sum_{n=-\infty}^{\infty} e\left(n^{2} z\right) \in \mathcal{M}_{1 / 2}\left(\Gamma_{0}(4), m_{\theta}\right)$.

The non-equivalent cusps of $\Gamma_{0}(4) \backslash \mathbb{H}$ are $i \infty, 0$ and $1 / 2$. As $\theta$ is not cuspidal at $i \infty$ or 0 and cuspidal at $1 / 2$, Corollary 2.2.4 (with $\alpha=1, r=1 / 2$ ) implies that $F(x)$ is differentiable in the orbit of $1 / 2=\{a /(2 b): 2 \nmid a, b\}[6]$ and non-differentiable elsewhere [7. By Corollary 2.2.3, the value of the derivative (when it exists) is $-\pi$ [6], and by Theorem 2.3 the fractal dimension of its graph [3] is $5 / 4$.

\section{§3. Some auxiliary Results}

In this section we shall state and prove some results that will be used in the next section. Here and hereafter we shall refer to the identity

$$
\int_{0}^{\infty} t^{\alpha-1} e^{-2 \pi n t} d t=\frac{\Gamma(\alpha)}{(2 \pi n)^{\alpha}}
$$

as the "gamma integral", and it will be a basic tool to introduce the weight $n^{-\alpha}$ in the coefficients of an automorphic form.

Our first result determines the ranges in which the definition of $f_{\alpha}$ makes sense.

Proposition 3.1. Let $\sigma_{\alpha}$ be the series defining $f_{\alpha}$.

a) If $f \in \mathcal{S}_{r}-\{0\}$, then $\sigma_{\alpha}$ converges to a continuous function for $\alpha>r / 2$ and diverges in a dense set for $\alpha \leq r / 2$.

b) If $f \in \mathcal{M}_{r}-\mathcal{S}_{r}$, then $\sigma_{\alpha}$ converges to a continuous function for $\alpha>r$ and diverges in a dense set for $\alpha \leq r$.

The main ingredient of the proof is the following result. For convenience, a few necessary but straightforward consequences of the expansion at the cusps (Lemma 3.3 and Lemma 3.5) are stated later.

Lemma 3.2 (cf. [10]). Let $f(z)=\sum a_{n} e(n z) \in \mathcal{M}_{r}-\{0\}$. For $C_{1}, C_{2}$ depending only on $f$ and every $N \geq 2$,

a) if $f \in \mathcal{S}_{r}$, then $\sum_{n \leq N} a_{n} e(n x)=O\left(N^{r / 2} \log N\right)$ uniformly in $x$;

b) if $f \in \mathcal{M}_{r}-\mathcal{S}_{r}$, then $\sum_{n \leq N} a_{n} e(n x)=O\left(N^{r} \log N\right)$ uniformly in $x$;

c) if $f \in \mathcal{S}_{r}$, then $C_{1} N^{r}<\sum_{n \leq N}\left|a_{n}\right|^{2}<C_{2} N^{r}$;

d) if $f \in \mathcal{M}_{r}-\mathcal{S}_{r}$, then $C_{1} \phi_{r}(N)<\sum_{n \leq N}\left|a_{n}\right|^{2}<C_{2} \phi_{r}(N)$, where

$$
\phi_{r}(N)=\left\{\begin{array}{lc}
N^{r} & \text { if } 0<r<1, \\
N \log N & \text { if } r=1, \\
N^{2 r-1} & \text { if } r>1 .
\end{array}\right.
$$


Proof. a) (Th. 5.3 of [10]). The function $g(z)=|\Im z|^{r / 2}|f(z)|$ is well defined in $\Gamma \backslash \mathbb{H}$, i.e., $g(\gamma z)=g(z)$ for $\gamma \in \Gamma$, and tends to zero at the cusps; hence $g$ is bounded. Let $D_{N}(z)=\sum_{|n| \leq N} e(n z)$, the Dirichlet kernel. Then

$$
\left|\sum_{n \leq N} a_{n} e(n x)\right| \leq \int_{0}^{1} N^{r / 2} g(u+i / N)\left|D_{N}(x-u-i / N)\right| d u \leq C N^{r / 2} \log N .
$$

b) The proof is like that of a) but using $|f(u+i / N)|<C N^{r}$, which is a consequence of the expansion at the cusps after Farey subdivision of level $N$ (see Lemma 3.5 below and [8]).

c) Let $g$ be the function defined in a). Consider the intervals $|x-a / b| \leq$ $1 /\left(b N^{1 / 2}\right)$, with $C_{1} N^{1 / 2}<b<C_{2} N^{1 / 2}$; by the expansion at the cusp $a / b$, there exists a constant $C>0$ such that $|\{x: g(x+i / N)>C\}|>0$. As $g$ is also bounded from above, by Parseval's identity

$$
K_{1} N^{r}<N^{r} \int_{0}^{1}|g(u+i / N)|^{2} d u=\sum_{n}\left|a_{n}\right|^{2} e^{-4 \pi n / N}<K_{2} N^{r} .
$$

The upper bound implies at once

$$
\sum_{n \leq N}\left|a_{n}\right|^{2}<C_{2} N^{r}
$$

On the other hand,

$$
\sum_{n \leq K N}\left|a_{n}\right|^{2} \geq \sum_{n}\left|a_{n}\right|^{2} e^{-4 \pi n / N}-\sum_{n>K N}\left|a_{n}\right|^{2} e^{-4 \pi n / N},
$$

which combined with the lower bound and (3.1) gives

$$
\sum_{n \leq K N}\left|a_{n}\right|^{2} \geq K_{1} N^{r}-C_{2} e^{-4 \pi K} N^{r}
$$

Choosing $K$ large enough, we get the result. (A more precise estimate can be obtained with less elementary arguments involving Rankin-Selberg convolution.)

d) The interval $[0,1]$ is covered by the union of

$$
J(A / B, k)=\left\{x: \frac{k-1}{N} \leq\left|x-\frac{A}{B}\right| \leq \frac{k}{N}\right\},
$$

where $1 \leq k \leq N^{1 / 2} / B$ and $A / B$ is irreducible, $0 \leq A \leq B \leq N^{1 / 2}$.

For each $x \in J(A / B, k)$ with $k>1$, let $a / b$ be the nearest Farey fraction of level $2 N /(k B)$. As $|x-A / B| \geq(k-1) / N$, by well known approximation properties (see Th. 36 of [8]), we have $|x-a / b|<k B /(2 N b) \leq b^{-2}$ and $a / b \neq A / B$, which combined with $|x-A / B| \leq k / N$ and $|a / b-A / B| \geq 1 /(b B)$ gives $b \geq C N /(k B)$. Hence Lemma 3.5 implies that

$$
|f(x+i / N)| \leq C\left(B^{r} k^{r}+N^{r} B^{-r} k^{-r}\right) \quad \text { for } x \in J(A / B, k),
$$

and we obtain

$$
\int_{0}^{1}|f(x+i / N)|^{2} d x \leq C \sum_{A, B, k}\left(B^{2 r} k^{2 r} N^{-1}+N^{2 r-1} B^{-2 r} k^{-2 r}\right) \leq K_{2} \phi_{r}(N) .
$$

For the lower bound, note that, as we mentioned in c), if

$$
g(x+i / N)=N^{-r / 2}|f(x+i / N)|>C
$$


on a set of positive measure, then

$$
K_{1} \phi_{r}(N) \leq \int_{0}^{1}|f(x+i / N)|^{2} d x \quad \text { for } 0<r<1 .
$$

The right hand side also is greater than

$$
\sum_{A, B} \int_{J(A / B, 1)}|f(x+i / N)|^{2} d x \geq C \sum_{A, B} N^{2 r-1} B^{-2 r} .
$$

Here we have used the expansion at the cusps (in the form of Lemma 3.3), and the summation is restricted to $0 \leq A \leq B \leq C^{\prime} N^{1 / 2}$, with $A / B$ irreducible and equivalent to a cusp in which $f$ is not cuspidal. As $\left[S L_{2}(\mathbf{Z}): \Gamma\right]<\infty$, the latter condition can be ruled out, keeping a comparable value of the sum (there are finitely many non-equivalent cusps $A / B)$. Hence we have proved

$$
\int_{0}^{1}|f(x+i / N)|^{2} d x \geq K_{1} \phi_{r}(N) \quad \text { for } r \geq 1 .
$$

We deduce from (3.2), (3.3) and (3.4) that

$$
K_{1} \phi_{r}(N) \leq \sum_{n}\left|a_{n}\right|^{2} e^{-4 \pi n / N} \leq K_{2} \phi_{r}(N)
$$

and the proof is completed as in c).

Our next result is a simple consequence of the expansion of $f$ at a cusp.

Lemma 3.3. Let $f \in \mathcal{M}_{r}$ and $z=x+i y \in \mathbb{H}$. If $|b z-a|^{2} / y<\delta$ for $a, b$ relatively prime integers, then

$$
|f(z)|<C(\delta)|b z-a|^{-r} e^{-K y|b z-a|^{-2}},
$$

and if $|b z-a|^{2} / y \rightarrow 0$, then

$$
|f(z)||b z-a|^{r} e^{K y|b z-a|^{-2}} \rightarrow C_{1},
$$

where $C_{1}>0$ and $K \geq 0$ depend only on the equivalence class of the cusp $\mathfrak{a}=a / b$, and $K=0$ if and only if $f$ is not cuspidal at $\mathfrak{a}$.

Proof. Let $\sigma_{\mathfrak{a}}$ be the scaling matrix for $\mathfrak{a}$; then $\sigma_{\mathfrak{a}}^{-1}(\mathfrak{a})=\infty$ and we can write

$$
\sigma_{\mathfrak{a}}^{-1}=\left(\begin{array}{cc}
C & 0 \\
0 & 1 / C
\end{array}\right) \gamma \quad \text { with } \gamma=\left(\begin{array}{cc}
* & * \\
b & -a
\end{array}\right) \in S L_{2}(\mathbf{Z})
$$

and $C \neq 0$ depending only on the equivalence class of $\mathfrak{a}$. Hence

$$
\Im \sigma_{\mathfrak{a}}^{-1}(z)=\frac{\Im z}{\left|j_{\sigma_{\mathfrak{a}}^{-1}}(z)\right|^{2}}=\frac{C^{2} y}{|b z-a|^{2}},
$$

and the lemma follows from the expansion of $f$ at the cusp $\mathfrak{a}$ (see $\S 2$ ) upon replacing $z$ by $\sigma_{\mathfrak{a}}^{-1}(z)$. (Note that $\left.j_{\sigma_{\mathfrak{a}}}\left(\sigma_{\mathfrak{a}}^{-1}(z)\right)=\left(j_{\sigma_{\mathfrak{a}}^{-1}}(z)\right)^{-1}\right)$.

In the following two lemmas we shall use generalised Ford circles (Speiser circles)

$$
\left\{z:\left|z-\frac{a}{c}-\frac{i}{t c^{2}}\right| \leq \frac{1}{t c^{2}}\right\}=\gamma\left(\left\{\Im z \geq \frac{t}{2}\right\}\right), \gamma=\left(\begin{array}{ll}
a & * \\
c & *
\end{array}\right) \in S L_{2}(\mathbf{Z}), c \neq 0, t>0 .
$$

Let us denote each of these circles by $\mathcal{F}_{a / c}$. They have the following elementary properties: For $t=1, \cup \mathcal{F}_{a / c} \supset\{0<\Im z<1 / 2\}$ because the standard fundamental domain of $S L_{2}(\mathbf{Z}) \backslash \mathbb{H}$ is contained in $\{\Im z \geq 1 / 2\}$. For $t=2$ (Ford circles) they form 
a set of mutually tangent circles in such a way that $\mathcal{F}_{a / c}$ is tangent to $\mathcal{F}_{a^{\prime} / c^{\prime}}$ if and only if $a / c$ and $a^{\prime} / c^{\prime}$ are consecutive Farey functions. For $t=\sqrt{5}$, Hurwitz' theorem in diophantine approximation (see Th. 193 of [8]) assures that the projections of the $\mathcal{F}_{a / c}$ on the real axis cover each irrational number infinitely many times; in fact, Hurwitz' theorem itself can be proved using the geometry of Ford circles (see [5]).

In general, there are striking relations between this and other generalizations of Ford circles, geodesics in Riemann surfaces and results in diophantine approximations (see [12]).

Lemma 3.4. Let $f \in \mathcal{M}_{r}-\{0\}$ and $x_{0} \in \mathbf{R}$. Then

$$
\varlimsup_{y \rightarrow 0^{+}} y^{r / 2}\left|f\left(x_{0}+i y\right)\right| \neq 0 \Leftrightarrow f \text { is not cuspidal at } x_{0},
$$

and this upper limit is finite everywhere for $f \in \mathcal{S}_{r}$.

Proof. The last part of the lemma is just a consequence of the properties of the function $g$, introduced in the proof of Lemma $3.2 \mathrm{a}$ ), and the first part is trivial for $x_{0} \in \mathbf{Q}$ because of the expansion at the cusps (see Lemma 3.3). Hence it remains only to prove that the upper limit does not vanish for $x_{0} \notin \mathbf{Q}$.

Let $\gamma_{1}^{-1}, \gamma_{2}^{-1}, \ldots$ be representatives of $S L_{2}(\mathbf{Z}) / \Gamma$. Then a fundamental domain $\mathcal{D}$ of $\Gamma \backslash \mathbb{H}$ can be constructed applying the $\gamma_{i}$ 's to the standard one of $S L_{2}(\mathbf{Z}) \backslash \mathbb{H}$. As the number of zeros of $f$ in $\mathcal{D}$ is finite, we can choose $2<t_{0}<\sqrt{5}$ such that $|f(z)| \geq C>0$ for $z \in \mathcal{D} \cap \bigcup \gamma_{i}\left(\left\{\Im z=t_{0} / 2\right\}\right)$. As the line $\left\{\Re z=x_{0}\right\}$ cuts infinitely many of the generalised Ford circles for $t \leq \sqrt{5}$, we can find $z=x_{0}+i y$ with $y$ arbitrarily small such that $z=\gamma\left(\sigma_{0}+i t_{0} / 2\right)$ with $\left|\sigma_{0}\right| \leq 1 / 2, \gamma \in S L_{2}(\mathbf{Z})$. Writing $\gamma^{-1}=\gamma_{i}^{-1} \tilde{\gamma}$ with $\tilde{\gamma} \in \Gamma$, we get

$$
\begin{aligned}
\left|f\left(x_{0}+i y\right)\right| & =\left|f\left(\gamma_{i}\left(\sigma_{0}+i t_{0} / 2\right)\right)\right|\left|j_{\gamma_{i} \gamma^{-1}}(z)\right|^{-r} \\
& \geq C\left|j_{\gamma_{i}}\left(\sigma_{0}+i t_{0} / 2\right)\right|^{-r}\left|j_{\gamma^{-1}}(z)\right|^{-r} \\
& \geq C^{\prime}\left|j_{\gamma^{-1}}(z)\right|^{-r}=C^{\prime} t_{0}^{r / 2}(2 y)^{-r / 2}, \quad C^{\prime}>0,
\end{aligned}
$$

and the result follows.

Lemma 3.5. If $|x-a / b| \leq b^{-2}$ with $a / b$ an irreducible fraction, then

$$
|f(x+i y)|<C\left(b^{-r} y^{-r}+b^{r}\right)
$$

Proof. Consider Ford circles for $t=1$. Let $\mathcal{F}_{A / B}$ be the circle containing $z=x+i y$ (we assume $0<y<1 / 2$ ). Note that

$$
\left|z-\frac{A}{B}-\frac{i}{B^{2}}\right| \leq \frac{1}{B^{2}} \Rightarrow y \leq \frac{2}{B^{2}}, \quad\left|x-\frac{A}{B}\right|^{2} \leq 2 y \Rightarrow \frac{|B z-A|^{2}}{y} \leq 4,
$$

and by Lemma 3.3 we obtain

$$
|f(x+i y)| \leq K\left((B x-A)^{2}+B^{2} y^{2}\right)^{-r / 2}
$$

If $B<b / 2$, then

$$
\left|x-\frac{A}{B}\right| \geq\left|\frac{A}{B}-\frac{a}{b}\right|-\left|x-\frac{a}{b}\right| \geq \frac{1}{b B}-\frac{1}{b^{2}}>\frac{1}{2 b B}
$$

and (3.5) gives $|f(x+i y)| \leq K(2 b)^{r}$. On the other hand, if $B \geq b / 2$ we obtain $|f(x+i y)| \leq K B^{-r} y^{-r} \leq K(b y / 2)^{-r}$ from (3.5). 
After these lemmas, Proposition 3.1 follows by standard arguments.

Proof (of Proposition 3.1). By Lemma $3.2 \mathrm{a}$ ), b) and partial summation it is plain that $\sigma_{\alpha}$ converges for $\alpha>r / 2$ when $f \in \mathcal{S}_{r}$ and for $\alpha>r$ when $f \in \mathcal{M}_{r}-\mathcal{S}_{r}$. It is also easy to prove that the function obtained is continuous (this can be deduced from Th. XII.9.1 of [17]).

Let us prove first that $\sigma_{r / 2}(x)$ diverges almost everywhere. Considering the kernels of summability $\varphi_{1}(u)=e^{-2 \pi u}\left(u^{r / 2}+1\right)$ and $\varphi_{2}(u)=e^{-2 \pi u}$, we have (see Th. III.1.2 of [17])

$$
\lim _{t \rightarrow 0^{+}} t^{r / 2} f(x+i t)=\lim _{t \rightarrow 0^{+}}\left(\sum \frac{a_{n}}{n^{r / 2}} e(n x) \varphi_{1}(n t)-\sum \frac{a_{n}}{n^{r / 2}} e(n x) \varphi_{2}(n t)\right)=0
$$

if $\sigma_{r / 2}(x)$ converges; but Lemma 3.4 (see above) implies that this only holds in, at most, a countable set of values of $x$.

Finally we shall prove that $\sigma_{r}(x)$ does not converge at every cusp $x_{0}$ at which $f$ is not cuspidal. If $\sigma_{r}\left(x_{0}\right)$ converges to $\ell$, by the gamma integral,

$$
\ell=\lim _{u \rightarrow 0^{+}} \sum \frac{a_{n}}{n^{r}} e\left(n x_{0}\right) e^{-2 \pi n u}=\frac{(2 \pi)^{r}}{\Gamma(r)} \lim _{u \rightarrow 0^{+}} \int_{u}^{\infty}(t-u)^{r-1}\left(f\left(x_{0}+i t\right)-a_{0}\right) d t .
$$

But the expansion at the cusp

$$
f\left(x_{0}+i t\right)=C_{1} t^{-r}+O\left(e^{-C_{2} / t}\right)
$$

and the divergence of the integral

$$
\int_{u}^{1}(t-u)^{r-1} t^{-r} d t
$$

as $u \rightarrow 0^{+}$imply $\ell=\infty$

Finally, we present some lemmas of an analytic nature.

Lemma 3.6. Let $g$ be a 1-periodic continuous function and let $G_{r}=G_{r}(x)$ be its Poisson integral. If $g \in \lambda_{\beta}\left(x_{0}\right), 0<\beta<1$, then for any positive integer $m$,

$$
\lim _{r \rightarrow 1^{-}}(1-r)^{m-\beta} G_{r}^{(m)}\left(x_{0}\right)=0 .
$$

Proof. The result is a simple generalization of Lemma 2.11 of [7] that can be obtained from the proof given there by just replacing the estimates for $P_{r}^{\prime}(t)$ by those for $P_{r}^{(m)}(t)$ (see (3.9) below).

Lemma 3.7. For $g:[0,1] \rightarrow \mathbf{R}$ continuous and non-constant, let $\mathcal{G}$ be its graph and define $G:[0,1] \rightarrow \mathbf{R}$ as

$$
G(x)=\int_{0}^{1-x}|g(x+t)-g(t)| d t .
$$

a) If $g \in \Lambda_{\beta}$ for every $\beta<\beta_{0}$, then

$$
\overline{\operatorname{dim}}(\mathcal{G}) \leq 2-\beta_{0} .
$$

b) If $\lim _{x \rightarrow 0^{+}} G(x) x^{-\beta}=\infty$ for every $\beta>\beta_{1}$, then

$$
\underline{\operatorname{dim}}(\mathcal{G}) \geq 2-\beta_{1} .
$$


Proof. The proof of a) is well known (see, for instance, Th. 8.1 of [4]). For b) note first that

$$
\sup _{x, y \in[j h,(j+2) h]}|g(x)-g(y)| \geq \frac{1}{h} \int_{j h}^{(j+1) h}|g(x+h)-g(x)| d x,
$$

and the supremum is less than or equal to the sum of the suprema over $[j h,(j+1) h]$ and $[(j+1) h,(j+2) h]$. Hence, substituting in the definition of $\operatorname{dim}(\mathcal{G})$, we get

$$
\underline{\operatorname{dim}}(\mathcal{G}) \geq \lim _{h \rightarrow 0^{+}} \frac{\log \left(h^{-2} G(h)\right)}{\log h} \geq 2-\beta,
$$

and the result follows upon letting $\beta \rightarrow \beta_{1}^{+}$.

The next results are sharper tools to calculate the dimension.

Proposition 3.8. Let $g$ be a continuous function, $g:[0,1] \rightarrow \mathbf{R}$ and $p \geq 1$. If $g=\sum g_{n}$ with $g_{n} \in W^{2, p}$, then

$$
V(g, h) \leq C \sum_{n} \min \left(T_{p}\left(g_{n}^{\prime}\right), h^{-1} T_{p}\left(g_{n}\right)\right),
$$

where $T_{p}(u)=\|u\|_{p}+h^{1 / p}\|u\|_{p}^{1 / p^{\prime}}\left\|u^{\prime}\right\|_{p}^{1 / p}, 1 / p+1 / p^{\prime}=1$.

Proof. By density (Th. VIII.6 [1]) we can assume that $g_{n}$ is differentiable. By the mean value theorem we have

$$
V\left(g_{n}, h\right)=h \sum_{j}\left|g_{n}^{\prime}\left(\xi_{j}\right)\right|, \quad(j-1) h<\xi_{j}<j h .
$$

Sobolev's inequality (cf. (29) on p. 168 of [1] ) applied to $\left(g_{n}^{\prime}\right)^{p}$ implies that

$$
\left|g_{n}^{\prime}\left(\xi_{j}\right)\right|^{p} \leq \frac{1}{|I|} \int_{I}\left|g_{n}^{\prime}\right|^{p}+p \int_{I}\left|g_{n}^{\prime}\right|^{p-1}\left|g_{n}^{\prime \prime}\right|
$$

for any interval $I \subset[0,1]$. Choosing $I=I_{j}=[(j-1) h, j h]$, summing over $j$ and applying Hölder's inequality, we obtain

$$
\left|g_{n}^{\prime}\left(\xi_{j}\right)\right|^{p} \leq h^{-1}\left\|g_{n}^{\prime}\right\|_{p}^{p}+p\left\|g_{n}^{\prime}\right\|_{p}^{p-1}\left\|g_{n}^{\prime \prime}\right\|_{p} \leq C h^{-1}\left|T_{p}\left(g_{n}^{\prime}\right)\right|^{p} .
$$

Hence

$$
V\left(g_{n}, h\right) \leq\left(h \sum_{j}\left|g_{n}\left(\xi_{j}^{\prime}\right)\right|^{p}\right)^{1 / p} \leq C^{\prime} T_{p}\left(g_{n}^{\prime}\right)
$$

Trivially we have

$$
V\left(g_{n}, h\right) \leq 2 \sum_{j}\left|g_{n}\left(\xi_{j}^{\prime}\right)\right|, \quad \xi_{j}^{\prime} \in I_{j} .
$$

The same argument as before proves

$$
V\left(g_{n}, h\right) \leq 2 h^{-1}\left(h \sum_{j}\left|g_{n}\left(\xi_{j}^{\prime}\right)\right|^{p}\right)^{1 / p} \leq C^{\prime \prime} T_{p}\left(g_{n}\right),
$$

and the result follows from (3.6) and (3.7) upon summing over $n$. 
Corollary 3.9. Let $g:[0,1] \rightarrow \mathbf{R}$ be continuous and let $\mathcal{G}$ be its graph. If the Fourier coefficients, $c_{k}$, of $g$ satisfy

$$
\sum_{N \leq|k|<2 N}\left|c_{k}\right|^{2}=O\left(N^{-\beta}\right), \quad \text { with } \beta>1,
$$

then

$$
\overline{\operatorname{dim}}(\mathcal{G}) \leq \max (1,2-\beta / 2) .
$$

Remark. Note that this result can be employed to obtain the upper bound in Theorem 3.1 of [3].

Proof. Simply choose $g_{0} \equiv c_{0}$ and

$$
g_{N}(x)=\sum_{N \leq|k|<2 N} c_{k} e(k x) \text { with } N=2^{n} .
$$

Parseval's identity implies that

$$
T_{2}\left(g_{N}^{\prime}\right)=O\left(N^{1-\beta / 2}+h^{1 / 2} N^{3 / 2-\beta / 2}\right)
$$

and

$$
T_{2}\left(g_{N}\right)=O\left(N^{-\beta / 2}+h^{1 / 2} N^{1 / 2-\beta / 2}\right) .
$$

The result is now a consequence of Proposition 3.8 (using the first bound for $1 \leq$ $N \leq h^{-1}$ and the second one for $N>h^{-1}$ ).

Proposition 3.10. Let $g$ be a 1-periodic continuous real function, $\mathcal{G}$ its graph (restricted to $[0,1])$ and $G_{r}(x)$ its Poisson integral. If for each $h \in(0,1)$ there exist $x_{1}, x_{2}, \ldots, x_{N} \in[0,1]$ with $x_{j+1}-x_{j} \geq h$ and $N>C_{1} h^{-\beta_{1}}>1$ such that

$$
h^{m-\beta_{2}}\left|G_{1-h}^{(m)}\left(x_{j}\right)\right|>C_{2}>0, \quad j=1,2, \ldots, N,
$$

for some positive integer $m$, then

$$
\underline{\operatorname{dim}}(\mathcal{G}) \geq \max \left(1,1+\beta_{1}-\beta_{2}\right) .
$$

Proof. By the Poisson integral representation we get

$$
\left|\int_{x_{j}-1 / 2}^{x_{j}+1 / 2}\left(g(x)-g\left(x_{j}\right)\right) P_{1-h}^{(m)}\left(x_{j}-x\right) d x\right|>C_{2} h^{-m+\beta_{2}},
$$

where $P_{r}(x)=\sum r^{|n|} e(n x)$ is the Poisson kernel (note that $\int P_{r}^{(m)}=0$ ).

The explicit formula for $P_{r}$ implies the bounds

$$
\begin{gathered}
\left|P_{r}^{(m)}(x)\right|=O\left((1-r) x^{-m-2}\right) \quad \text { for } \quad|x|>1-r, \\
\left|P_{r}^{(m)}(x)\right|=O\left((1-r)^{-m-1}\right) \quad \text { for } \quad|x| \leq 1-r .
\end{gathered}
$$

Consider the intervals

$$
J_{-1}\left(x_{j}\right)=\emptyset, J_{k}\left(x_{j}\right)=\left(-2^{k} h+x_{j}, x_{j}+2^{k} h\right), k=0,1,2, \ldots
$$

Dyadic subdivision in (3.8) implies that we can find $k_{j}$ and $y_{j} \in J_{k_{j}}\left(x_{j}\right)-J_{k_{j}-1}\left(x_{j}\right)$ such that

$$
2^{k_{j}} h\left|g\left(y_{j}\right)-g\left(x_{j}\right)\right|\left|P_{1-h}^{(m)}\left(x_{j}-y_{j}\right)\right|>C_{3} h^{-m+\beta_{2}+\varepsilon}
$$

for any $\varepsilon>0$.

For the sake of brevity we shall write $T_{j}$ instead of $J_{k_{j}}\left(x_{j}\right)$. Using (3.9), the last inequality gives

$$
\left|T_{j}\right| \cdot\left|g\left(y_{j}\right)-g\left(x_{j}\right)\right| \cdot h\left|T_{j}\right|^{-m-2}>K_{\varepsilon} h^{-m+\beta_{2}+\varepsilon} .
$$


In particular, using $\left|T_{j}\right|^{-m}<h^{-m}$, we get

$$
V\left(g, h ; T_{j}\right)>K_{\varepsilon} h^{\beta_{2}-1+\varepsilon}\left|T_{j}\right| .
$$

As $T_{j} \supset\left(x_{j}-h / 2, x_{j}+h / 2\right)$,

$$
\left|\bigcup_{j} T_{j}\right| \geq N h>C_{1} h^{1-\beta_{1}} .
$$

By a covering lemma (see Lemma 7.3 of [13]) we can choose a subset $\left\{T_{j_{n}}\right\} \subset\left\{T_{j}\right\}$ such that the $T_{j_{n}}$ 's are disjoint and satisfy

$$
\left|\bigcup_{n} T_{j_{n}}\right|=\sum_{n}\left|T_{j_{n}}\right| \geq C_{1}^{\prime} h^{1-\beta_{1}} .
$$

Summing in (3.10) over $j=j_{n}$, we finally obtain

$$
V(g, h)>K_{\varepsilon}^{\prime} h^{\beta_{2}-\beta_{1}+\varepsilon}
$$

for any $\varepsilon>0$, and the lower bound for the dimension follows.

\section{§4. Proof of the main Results}

Proof of Theorem 2.1. If $f$ is a cusp form, it is easy to deduce from the estimates for its Fourier coefficients that $f_{\alpha} \in \Lambda_{\alpha-r / 2}^{\log }, 0<\alpha-r / 2<1$. Namely, given $0<h<1$ and $N>1$, we can write

$$
\begin{aligned}
\left|f_{\alpha}(x+h)-f_{\alpha}(x)\right| & \leq\left|\sum_{n<N} \frac{a_{n}}{n^{\alpha}} e(n x)(e(n h)-1)\right|+\left|\sum_{n \geq N} \frac{a_{n}}{n^{\alpha}}(e(n(x+h))-e(n x))\right| \\
& =\left|S_{1}\right|+\left|S_{2}\right| .
\end{aligned}
$$

Applying the mean value theorem to the real and imaginary parts of $S_{1}$, we have

$$
\left|S_{1}\right| \leq 4 \pi h\left|\sum_{n<N} \frac{a_{n}}{n^{\alpha-1}} e(n(x+\xi))\right|
$$

for some $\xi \in(0, h)$. Using the estimate of the proof of Lemma 3.2 a) and applying partial summation to the latter inequality and to $S_{2}$, we find that

$$
\left|f_{\alpha}(x+h)-f_{\alpha}(x)\right| \leq C\left(h N^{r / 2-\alpha+1}+N^{r / 2-\alpha}\right) \log N .
$$

Finally, choosing $N=h^{-1}$, we get that $f_{\alpha} \in \Lambda_{\alpha-r / 2}^{\log }$.

Note that it remains only to prove that $f_{\alpha} \notin \lambda_{\alpha-r / 2}\left(x_{0}\right)$ if $f$ is not cuspidal at $x_{0}$. Our arguments can be considered an extension of [7].

Let $m=[\alpha]+1$, where $[\cdot]$ indicates the integral part, and let

$$
F(z)=\sum a_{n} n^{m-\alpha} e(n z) \text {. }
$$

If $f_{\alpha} \in \lambda_{\alpha-r / 2}\left(x_{0}\right)$, by Lemma 3.6 with $r=e^{-2 \pi y}$ we have

$$
\lim _{y \rightarrow 0^{+}}\left(1-e^{-2 \pi y}\right)^{m-\alpha+r / 2} F\left(x_{0}+i y\right)=0 ;
$$

hence for $\varepsilon>0$ there exists $\delta>0$ such that

$$
\left|F\left(x_{0}+i y\right)\right|<\varepsilon y^{\alpha-m-r / 2}
$$

for $0<y<\delta$.

Using the gamma integral, we get

$$
y^{r / 2} f\left(x_{0}+i y\right)=a_{0} y^{r / 2}+\frac{(2 \pi)^{m-\alpha}}{\Gamma(m-\alpha)} \int_{0}^{\infty} t^{m-\alpha-1} y^{r / 2} F\left(x_{0}+i(t+y)\right) d t .
$$


By (4.1), if $y<\delta / 2$, the contribution to the integral in the range $0<t<\delta / 2$ is bounded by $C \varepsilon$. Trivially, the contribution of $t \geq \delta / 2$ is less than $C_{\delta} y^{r / 2}$. Hence we have proved that

$$
\varlimsup_{y \rightarrow 0^{+}} y^{r / 2}\left|f\left(x_{0}+i y\right)\right|<C \varepsilon,
$$

which contradicts Lemma 3.4 upon choosing $\varepsilon$ arbitrarily small.

Corollary 2.1.1 is a trivial consequence of the theorem because there are no irrational cusps.

Proof of Theorem 2.2. By Theorem 2.1, if $f$ is not cuspidal at $x_{0}$, then $f_{\alpha} \notin$ $\lambda_{\alpha-r / 2}\left(x_{0}\right)$; hence we have to consider only the case in which $f$ is cuspidal at $x_{0}$.

Let $0<h<1$ (the case with negative $h$ is similar). By the gamma integral

$$
f_{\alpha}\left(x_{0}+h\right)-f_{\alpha}\left(x_{0}\right)=\frac{(2 \pi)^{\alpha}}{\Gamma(\alpha)} \int_{0}^{\infty} t^{\alpha-1}\left(f\left(x_{0}+h+i t\right)-f\left(x_{0}+i t\right)\right) d t=\frac{(2 \pi)^{\alpha}}{\Gamma(\alpha)} I .
$$

Let us divide the range of integration, obtaining three integrals over the intervals $\left[0, y_{1}\right],\left[y_{1}, y_{2}\right],\left[y_{2}, \infty\right)$ respectively. Applying the mean value theorem to the real and imaginary parts of the integrand of the last integral (regarded as a function of $h$ ) and choosing $y_{1}=h^{2}, y_{2}=h$, we obtain

$$
I=I_{1}+I_{2}+h\left(\Re I_{3}\left(\xi_{1}\right)+i \Im I_{3}\left(\xi_{2}\right)\right),
$$

where $0<\xi_{j}<h$ and

$$
\begin{gathered}
I_{1}=\int_{0}^{h^{2}} t^{\alpha-1}\left(f\left(x_{0}+h+i t\right)-f\left(x_{0}+i t\right)\right) d t, I_{2}=\int_{h^{2}}^{h} t^{\alpha-1}\left(f\left(x_{0}+h+i t\right)-f\left(x_{0}+i t\right)\right) d t, \\
I_{3}(\xi)=\int_{h}^{\infty} t^{\alpha-1} f^{\prime}\left(x_{0}+\xi+i t\right) d t .
\end{gathered}
$$

Note that the exponential decay at the cusp $x_{0}$ implies that the contribution of $f\left(x_{0}+i t\right)$ to $I_{1}$ and $I_{2}$ is negligible in comparison with $h$.

If $f \in S_{r}$, then by the second part of Lemma 3.4

$$
\int_{0}^{h^{2}} t^{\alpha-1}\left|f\left(x_{0}+h+i t\right)\right| d t<C \int_{0}^{h^{2}} t^{\alpha-r / 2-1} d t=O\left(h^{2 \alpha-r}\right) .
$$

Let $x_{1}, x_{2}$ be such that $x_{0}, x_{1}, x_{2}$ are consecutive Farey fractions with $x_{0}<$ $x_{1}<x_{0}+h \leq x_{2}$; then for $a / b=x_{1}$ or $x_{2}$ we have $\left|x_{0}+h-a / b\right| \leq b^{-2}$. Since $x_{1}-x_{0}<h$ and $x_{2}-x_{0} \geq h$, it is easy to deduce (use elementary properties of Farey fractions [8]) that $b=O\left(h^{-1}\right)$ and $h^{-1}=O(b)$, with an $O$-constant depending on the denominator of $x_{0}$. Hence by Lemma 3.5, for $f \in \mathcal{M}_{r}$,

$$
\int_{0}^{h^{2}} t^{\alpha-1}\left|f\left(x_{0}+h+i t\right)\right| d t<C \int_{0}^{h^{2}} t^{\alpha-1}\left(h^{r} t^{-r}+h^{-r}\right) d t=O\left(h^{2 \alpha-r}\right) .
$$

Therefore we have proved

$$
I_{1}=O\left(h^{2 \alpha-r}\right) .
$$

Taking $z=x_{0}+h+i t$ and $a / b=x_{0}$ in Lemma 3.3, we obtain $\left|f\left(x_{0}+h+i t\right)\right| \leq$ $C_{1} h^{-r} e^{-C_{2} t h^{-2}}$; hence we have also

$$
I_{2}=O\left(h^{2 \alpha-r}\right) .
$$


Let $g(z)=-i(i(\xi-z))^{\alpha-1} f^{\prime}\left(z+x_{0}\right)$ and let $\gamma_{1}, \gamma_{2}, \gamma_{3}$ be the straight lines forming the positively oriented border, $\partial D$, of $D=\{0 \leq \Re z \leq \xi$, $z z \geq h\}$ (with $\Re \gamma_{1}=0$ and $\Re \gamma_{3}=\xi$ ). Then we can write $I_{3}(\xi)$ as

$$
I_{3}(\xi)=\int_{\gamma_{3}} g(z) d z=-\int_{\gamma_{1}}-\int_{\gamma_{2}}+\int_{\partial D}=-J_{1}-J_{2}+J_{3} .
$$

Note that $J_{3}$ vanishes identically because $g$ is holomorphic in $D$ (and decays at infinity). On the other hand, integrating by parts and using Lemma 3.3 as before, we get $\left|J_{2}\right|<C_{1} h^{-r} e^{-C_{2} / h}$. Finally, using the Taylor expansion $(i(\xi-i t))^{\alpha-1}=$ $t^{\alpha-1}+O\left(\xi t^{\alpha-2}\right)$, we deduce that

$$
J_{1}=-\int_{h}^{\infty} t^{\alpha-1} f^{\prime}\left(x_{0}+i t\right) d t+O(h)=-\int_{0}^{\infty} t^{\alpha-1} f^{\prime}\left(x_{0}+i t\right) d t+O(h),
$$

and hence

$$
I_{3}(\xi)=\int_{0}^{\infty} t^{\alpha-1} f^{\prime}\left(x_{0}+i t\right) d t+O(h) .
$$

Substituting (4.3), (4.4) and (4.5) in (4.2), we obtain

$$
f_{\alpha}\left(x_{0}+h\right)-f_{\alpha}\left(x_{0}\right)=h \frac{(2 \pi)^{\alpha}}{\Gamma(\alpha)} \int_{0}^{\infty} t^{\alpha-1} f^{\prime}\left(x_{0}+i t\right) d t+O\left(h^{2 \alpha-r}\right)+O\left(h^{2}\right),
$$

and the result follows.

Corollary 2.2.1 is an immediate consequence of Theorem 2.2 because, by definition, a cusp form is cuspidal exactly at every cusp.

Proof of Corollary 2.2.2. By the gamma integral, for $s \in \mathbf{R}^{+}$

$$
L(f, s)=\frac{(2 \pi)^{s}}{\Gamma(s)} \int_{0}^{\infty} t^{s-1} f(i t) d t .
$$

Note that the integral converges (because $f \in S_{r}$ ) and gives an analytic continuation of $L(f, s)=\sum a_{n} n^{-s}$ over the whole complex plane (defining $1 / \Gamma(s)$ as zero for $s=0,-1,-2 \ldots)$.

Integrating by parts in the formula for $f_{\alpha}^{\prime}\left(x_{0}\right)$ and taking $x_{0}=0$, we get

$$
f_{\alpha}^{\prime}(0)=\frac{(2 \pi)^{\alpha}}{\Gamma(\alpha-1)} i \int_{0}^{\infty} t^{\alpha-2} f(i t) d t=2 \pi i L(f, \alpha-1) .
$$

By properties of Gauss sums,

$$
\sum_{a=1}^{b} \chi(a) f(z+a / b)=\tau(\chi) g(z),
$$

where $g=f_{\bar{\chi}}$. Hence

$$
\sum_{a=1}^{b} \chi(a) f_{\alpha}^{\prime}(a / b)=\tau(\chi) g_{\alpha}^{\prime}(0),
$$

and the result is a consequence of (4.6).

Corollary 2.2.3 is a particular case of the formula for $f_{\alpha}^{\prime}\left(x_{0}\right)$ because $f\left(x_{0}+i t\right) \rightarrow$ 0 as $t \rightarrow 0^{+}$and $f\left(x_{0}+i t\right) \rightarrow a_{0}$ as $t \rightarrow+\infty$. 
Proof of Corollary 2.2.4. Assume that $g_{\alpha}=\Re f_{\alpha}$, the case $g_{\alpha}=\Im f_{\alpha}$ being similar.

Trivially, by Theorem 2.2, if $f$ is cuspidal at $x_{0}$, then $g_{\alpha}$ is differentiable at $x_{0}$. On the other hand, if $g_{\alpha}$ is differentiable at $x_{0}$, then $g_{\alpha} \in \Lambda_{\beta}\left(x_{0}\right)$ for $\alpha-r / 2<\beta<1$, and the boundness of the conjugate operator in Lipschitz spaces (see the following remark and III.13.29 of [17]) implies that $\Im f_{\alpha} \in \Lambda_{\beta}^{\log }\left(x_{0}\right)$; hence $f_{\alpha} \in \lambda_{\alpha-r / 2}\left(x_{0}\right)$, and $f$ is cuspidal at $x_{0}$ by Theorem 2.1 .

Remark. Note that III.13.29 of [17] is stated in global form but, with the notation used there, the proof (via 13.30) can be extended to $f \in \Lambda_{\beta}\left(x_{0}\right) \cap \Lambda_{\varepsilon}$ simply by employing the estimate

$$
\int_{-2 h}^{2 h} \frac{|f(x+t)-f(x+h)|}{|t-h|} d t \leq C \int_{-2 h}^{2 h} \frac{\min \left(|t-h|^{\varepsilon},|t|^{\beta}+h^{\beta}\right)}{|t-h|} d t=O\left(h^{\beta}|\log h|\right)
$$

to bound the corresponding part of the integral defining $\tilde{f}(x+h)$.

In proving Theorem 2.3 we shall distinguish the cuspidal and the non-cuspidal cases because, as we mentioned before, the latter one requires finer considerations.

In both cases we shall assume in the proofs that $g_{\alpha}=\Re f_{\alpha}$. The case $g_{\alpha}=\Im f_{\alpha}$ is completely similar.

Proof of Theorem 2.3 (cuspidal case). By Theorem 2.1 we can choose $\beta_{0}=\alpha-r / 2$ in Lemma 3.7 a), obtaining, for $\alpha-r / 2<1$,

$$
\overline{\operatorname{dim}}(\mathcal{G}) \leq 2+r / 2-\alpha .
$$

As $g_{\alpha}$ is 1-periodic and continuous, with the notation of Lemma 3.7, for $g=g_{\alpha}$

$$
G(x)=\int_{0}^{1}|g(x+t)-g(t)| d t+O(x) .
$$

We have the trivial inequality

$$
\int_{0}^{1}|g(x+t)-g(t)| d t \geq \frac{\int_{0}^{1}|g(x+t)-g(t)|^{2} d t}{\sup _{t}|g(x+t)-g(t)|} .
$$

By Parseval's identity the numerator of the right hand side is

$$
4 \int_{0}^{1}\left|\sum \frac{a_{n}}{n^{\alpha}} \sin (\pi n x) \sin (2 \pi n(t+x / 2))\right|^{2} d t=2 \sum \frac{\left|a_{n}\right|^{2}}{n^{2 \alpha}} \sin ^{2}(\pi n x) .
$$

Hence, considering the contribution of the range $1<4 n x<3$ and using Lemma $3.2 \mathrm{c}$ ), we obtain

$$
\int_{0}^{1}|g(x+t)-g(t)|^{2} d t>C x^{2 \alpha-r} .
$$

On the other hand, by the mean value theorem the denominator in (4.8) can be written as

$$
\left|-2 \pi x \sum_{n<x^{-1}} \frac{a_{n}}{n^{\alpha-1}} \sin \left(2 \pi\left(\xi+t_{0}\right)\right)+\sum_{n \geq x^{-1}} \frac{a_{n}}{n^{\alpha}}\left(\cos \left(2 \pi n\left(x+t_{0}\right)\right)-\cos \left(2 \pi n t_{0}\right)\right)\right|,
$$

where $0<\xi<x$ and $t_{0}$ is the value at which the supremum is achieved. Then, by Lemma 3.2 a), for $\alpha-r / 2<1$

$$
\sup _{t}|g(x+t)-g(t)|=O\left(x^{\alpha-r / 2-\varepsilon}\right), \quad \forall \varepsilon>0 .
$$


Substituting these bounds in (4.8) and (4.7), we conclude by Lemma $3.7 \mathrm{~b}$ ) that

$$
\underline{\operatorname{dim}}(\mathcal{G}) \geq 2+r / 2-\alpha
$$

hence

$$
\operatorname{dim}(\mathcal{G})=2+r / 2-\alpha, \quad \text { for } \alpha-r / 2<1 .
$$

Note that for $\alpha-r / 2 \geq 1,(4.9)$ holds upon replacing $\alpha-r / 2-\varepsilon$ by $1-\varepsilon$, which immediately gives $\operatorname{dim}(\mathcal{G})=1$ by Lemma 3.7 a).

Proof of Theorem 2.3 (non-cuspidal case). a) The upper bound:

For $\alpha>1$, the upper bound

$$
\overline{\operatorname{dim}}(\mathcal{G}) \leq \max (1,2+r / 2-\alpha)
$$

is a direct consequence of Corollary 3.9 and Lemma $3.2 \mathrm{~d}$ ).

For $\alpha \leq 1$, we start by considering the (Dirichlet) covering of $[0,1]$ given by the intervals

$$
I_{a / b}=\left\{x:\left|x-\frac{a}{b}\right| \leq \frac{h^{1 / 2}}{b}\right\},
$$

where $a / b$ is an irreducible fraction with $0 \leq a \leq b \leq h^{-1 / 2}$.

Applying the mean value theorem as in (4.2), we have for $x, y \in I_{a / b}$ that

$$
\left|g_{\alpha}(x)-g_{\alpha}(y)\right| \leq C\left(\left|J_{1}\right|+h^{1 / 2} b^{-1}\left|J_{2}\right|\right),
$$

where

$$
J_{1}=\int_{0}^{h^{1 / 2} b^{-1}} t^{\alpha-1}(f(x+i t)-f(y+i t)) d t, \quad J_{2}=\int_{h^{1 / 2} b^{-1}}^{\infty} t^{\alpha-1} f^{\prime}(\xi+i t) d t
$$

for some $\xi \in I_{a / b}$.

Lemma 3.5 applied to $J_{1}$ and $J_{2}$, after partial integration, gives

$$
\left|g_{\alpha}(x)-g_{\alpha}(y)\right|=O\left(h^{(\alpha-r) / 2} b^{-\alpha}+h^{\alpha / 2} b^{r-\alpha}\right) .
$$

Hence

$$
\begin{aligned}
V\left(g_{\alpha}, h\right) & \leq \sum_{a, b} V\left(g_{\alpha}, h ; I_{a / b}\right) \\
& =O\left(\sum_{a, b}\left(h^{(\alpha-r-1) / 2} b^{-\alpha-1}+h^{(\alpha-1) / 2} b^{r-\alpha-1}\right)\right)=O\left(h^{\alpha-r / 2-1} \log h\right) .
\end{aligned}
$$

This proves the upper bound for $\alpha \leq 1$.

b) The lower bound:

Define $y=-\frac{1}{2 \pi} \log (1-h)$. Then $G_{r}(t)$, the Poisson integral of $g_{\alpha}$, satisfies

$$
G_{1-h}(x)=\Re f_{\alpha}(x+i y)
$$

(where $f_{\alpha}$ is extended to the upper half plane in the obvious way).

We shall apply Proposition 3.10 with $x_{j}=a_{j} / b_{j}+\tau_{j} y$, where $0 \leq \tau_{j}<1$ is to be chosen later and $a_{j} / b_{j}$ are reduced fractions, with $y^{-1 / 2+\varepsilon} \leq b_{j} \leq C y^{-1 / 2+\varepsilon}$, $0<\varepsilon<1 / 2$, at which $f$ is not cuspidal. It is plain that for $h$ small enough the fractions satisfy the spacing condition required in the lemma (because $b_{j}^{-2} \geq$ $C^{\prime} y^{1-2 \varepsilon}, 2 \pi y \sim h$ ), and we can take $\beta_{1}=1-2 \varepsilon$ (see the comments before (3.4) in the proof of Lemma 3.2). 
Assume first that $\alpha$ is integral and even (the odd case proceeds in the same way, but with $\Im$ replacing $\Re)$. Taking $m=\alpha$, we get

$$
\left|G_{1-h}^{(m)}\left(x_{j}\right)\right|=\left|\Re f\left(x_{j}+i y\right)\right| .
$$

The expansion at the cusp $a_{j} / b_{j}$ implies that, for some $\mu \in \mathbf{C}-\{0\}$,

$$
f\left(x_{j}+i y\right) \sim \mu\left(b_{j}\left(x_{j}+i y\right)-a_{j}\right)^{-r}=\mu\left(b_{j} y\right)^{-r}\left(\tau_{j}+i\right)^{-r}
$$

as $y \rightarrow 0^{+}$, and a suitable choice of $\tau_{j}$ gives

$$
\left|\Re f\left(x_{j}+i y\right)\right|>C y^{-r / 2-\varepsilon r} .
$$

Hence we can take $\beta_{2}=\alpha-r / 2-\varepsilon r$ in Proposition 3.10, getting

$$
\underline{\operatorname{dim}}(\mathcal{G}) \geq \max (1,2+r / 2-\alpha+(r+2) \varepsilon),
$$

and the result is obtained as $\varepsilon \rightarrow 0^{+}$.

If $\alpha$ is not integral and $[\alpha]+1$ is even (again, the odd case is completely similar), using the gamma integral we have for $m=[\alpha]+1$

$$
\left|G_{1-h}^{(m)}\left(x_{j}\right)\right|=\left|\Re I\left(x_{j}\right)\right|,
$$

where

$$
I\left(x_{j}\right)=\frac{(2 \pi)^{\beta}}{\Gamma(\beta)} \int_{0}^{\infty} t^{\beta-1} f^{\prime}\left(x_{j}+i t+i y\right) d t \quad \text { with } \beta=\alpha+1-m .
$$

Let $I_{1}$ and $I_{2}$ be the contributions to the integral for $0<t<T$ and $t>T$, respectively, i.e.,

$$
I\left(x_{j}\right)=\frac{(2 \pi)^{\beta}}{\Gamma(\beta)}\left(I_{1}+I_{2}\right) .
$$

Integrating $I_{2}$ by parts, we get

$$
I_{2}=i T^{\beta-1}\left(f\left(x_{j}+i y+i T\right)-a_{0}\right)+i(\beta-1) \int_{T}^{\infty} t^{\beta-2}\left(f\left(x_{j}+i t+i y\right)-a_{0}\right) d t .
$$

By Lemma 3.5 we have

$$
\left|f\left(x_{j}+i t+i y\right)-a_{0}\right|<C \min \left(b_{j}^{-r}(t+y)^{-r}+b_{j}^{r},(t+y)^{-r}\right),
$$

which when substituted into the preceding formula for $I_{2}$ with the choice $T=y^{1-2 \varepsilon}$ gives

$$
\left|I_{2}\right|=O\left(y^{\delta}\right)
$$

with $\delta=\beta-1-r / 2+\varepsilon(2+r-2 \beta)$.

To treat $I_{1}$, note that upon differentiating the expansion of $f$ at the cusp $a_{j} / b_{j}$ we obtain for $z=x_{j}+i y+i t, 0<t<T$,

$$
f^{\prime}(z)=-r \mu b_{j}\left(b_{j} z-a_{j}\right)^{-r-1}+O\left(\left|b_{j} z-a_{j}\right|^{-r-2} e^{-C(y+t)\left|b_{j} z-a_{j}\right|^{-2}}\right) .
$$

For $t$ far from $T$ (say $t<y^{1-\epsilon}$ ) the error term is exponentially small, and a calculation proves that its total contribution to $I_{1}$ is absorbed by $O\left(y^{\delta}\right)$. Hence

$$
\begin{aligned}
I_{1} & =-r \mu b_{j} \int_{0}^{T} t^{\beta-1}\left(b_{j} \tau_{j} y+b_{j}(y+t) i\right)^{-r-1} d t+O\left(y^{\delta}\right) \\
& =-r \mu b_{j}^{-r} y^{-r-1+\beta} \int_{0}^{y^{-2 \varepsilon}} u^{\beta-1}\left(\tau_{j}+i+i u\right)^{-r} d t+O\left(y^{\delta}\right) .
\end{aligned}
$$


With a suitable choice of $\tau_{j}$ we have

$$
\left|\Re I_{1}\right|>C y^{\beta-1-r / 2-r \varepsilon} .
$$

Substituting this and (4.12) into (4.11) and (4.10), we conclude that

$$
\left|G_{1-h}^{(m)}\left(x_{j}\right)\right|>C^{\prime} y^{\alpha-m-r / 2-r \varepsilon} .
$$

Hence we can take $\beta_{2}=\alpha-r / 2-\varepsilon r$ in Proposition 3.10, as in the case with $\alpha$ integral, and the proof is completed in the same way.

\section{$\S 5$. The theta Function CASE}

In this section we shall apply our results in the context of theta functions, which play an important role in the theory of automorphic forms, providing some simple and explicit examples.

Given a positive definite quadratic form in $\mathbf{R}^{k}$ with integral coefficients, $Q(\vec{x})=$ $\vec{x}^{t} M \vec{x}$, the corresponding theta function,

$$
\Theta(z)=\sum_{\vec{n} \in \mathbf{Z}^{k}} e(Q(\vec{n}) z),
$$

belongs to $\mathcal{M}_{k / 2}\left(\Gamma_{0}(2 N), m\right)$, where $N=\operatorname{det}(2 M)$ and $m$ is a certain multiplier system (see Th. 10.8 in [10]). The computation of its expansion at the cusp $\mathfrak{a}=a / b$ gives that $\Theta$ is cuspidal at $\mathfrak{a}$ if and only if

$$
S(a, b)=\sum_{\vec{n} \bmod b} e\left(\frac{a Q(\vec{n})}{b}\right)=0
$$

(see $\S 10.3$ in [10], especially 10.25), where $\vec{n} \bmod b$ means that each coordinate of $\vec{n}$ runs over a complete residue class modulo $b$.

Proposition 5.1. Let $r_{Q}(n)=\left|\left\{\vec{x} \in \mathbf{Z}^{k}: Q(\vec{x})=n\right\}\right|$ with $Q$ as before. Let $k / 2<\alpha<k / 2+1$ if $k>1$, and $3 / 4<\alpha<3 / 2$ if $k=1$. Then the function

$$
F(x)=\sum_{n=1}^{\infty} \frac{r_{Q}(n)}{n^{\alpha}} e(n x)
$$

is differentiable at $x_{0} \in \mathbf{Q}$ if and only if $S(1, b)=0$, where $b$ is the denominator of $x_{0}$ (written in lowest terms).

Using a well known formula for Gauss sums, we immediately deduce

Corollary 5.1.1. Let $r_{k}(n)$ be the number of representations of $n$ as a sum of $k$ squares. Then, for $\alpha$ as before, the function

$$
F(x)=\sum_{n=1}^{\infty} \frac{r_{k}(n)}{n^{\alpha}} e(n x)
$$

is differentiable at $x_{0} \in \mathbf{Q}$ if and only if $x_{0}=a /(2 b)$ with $a$ and $b$ odd integers.

Now we consider the special case of functions defined in Proposition 5.1 for $k=1$ and $k=2$. In both cases Theorem 2.3 applies, proving that the fractal dimension of their graphs is $\max (1,2-\alpha+k / 4)$.

Note that the simplest case, $k=1$, includes Riemann's example. To keep the analogy with Corollary 2.2.2, we shall express the value of the derivative at $1 / 2$ in terms of the Riemann $\zeta$-function. 
Proposition 5.2. Consider the function

$$
F(x)=\sum_{n=1}^{\infty} \frac{e\left(n^{2} x\right)}{n^{\beta}} .
$$

Then for $\beta>3 / 2$

$$
F^{\prime}(1 / 2)=2 \pi i\left(2^{3-\beta}-1\right) \zeta(\beta-2) .
$$

With a closer analysis of the binary case we shall show that a direct evaluation of $S(1, b)$ can be avoided in Proposition 5.1.

Proposition 5.3. Consider a binary integral definite positive quadratic form $Q(x, y)$ $=A x^{2}+B x y+C y^{2}$. Let $2^{r}$ and $2^{s}$ be the highest powers of 2 dividing the discriminant $D=B^{2}-4 A C$ and $\operatorname{gcd}(A, B, C)$, respectively; then the function

$$
F(x)=\sum_{n=1}^{\infty} \frac{r_{Q}(n)}{n^{\alpha}} e(n x), \quad 1<\alpha<3 / 2,
$$

(and its real and imaginary parts) is continuous but nowhere differentiable if and only if $r=2 s$. Otherwise, $F$ is differentiable at $x_{0}$ if and only if $x_{0}=2^{-s-1} a / b$ or $x_{0}=2^{1+s-r} a / b$ with $a$ and $b$ odd integers.

Examples. When $(n, m)$ runs over $\mathbf{Z}^{2}-\{(0,0)\}$, the function

$$
\sum_{n, m} \frac{e\left(\left(n^{2}+m n+m^{2}\right) x\right)}{\left(n^{2}+m n+m^{2}\right)^{5 / 4}}
$$

is nowhere differentiable because $r=s=0$, and

$$
\sum_{n, m} \frac{e\left(\left(n^{2}+2 m^{2}\right) x\right)}{\left(n^{2}+2 m^{2}\right)^{5 / 4}}
$$

is differentiable at $x_{0}=a / b$ if and only if $b \equiv 2,4,6$ (8) and $a$ is odd, because $r=3$, $s=0$.

Given a primitive character $\psi$ with conductor $|\psi|$ and $k$ a positive integer, the associated theta function

$$
\Theta(z, k, \psi)=\sum_{n=-\infty}^{\infty} \psi(n) e\left(k n^{2} z\right)
$$

belongs to $\mathcal{M}_{1 / 2}\left(\Gamma_{0}(4 N), \chi\right)$, where $N=k|\psi|^{2}$ and $\chi=\left(\frac{D}{.}\right) \psi$ with $D$ the discriminant of $\mathbf{Q}(\sqrt{k})$. An important result due to J.-P. Serre and H. Stark 14 asserts that, for any character $\chi, \mathcal{M}_{1 / 2}\left(\Gamma_{0}(4 N), \chi\right)$ is generated (as a vector space) by all of the theta functions of this type, and $S_{1 / 2}\left(\Gamma_{0}(4 N), \chi\right)$ is obtained if $\psi$ is restricted to have some odd $p$-part. (Note that we can assume that $\psi$ is globally even, i.e. $\psi(-1)=1$, because otherwise $\Theta(\cdot, k, \psi)$ vanishes identically.)

Examples. Consider $\psi=\chi_{3} \cdot \chi_{4}$, where $\chi_{3}$ and $\chi_{4}$ are the (only) primitive characters of conductor 3 and 4 , as they are not even. Then

$$
\Theta(z, 1, \psi)=\sum_{n=-\infty}^{\infty} \psi(n) e\left(n^{2} z\right) \in S_{1 / 2}\left(\Gamma_{0}(576), \psi\right) .
$$


By Corollary 2.2.1 and Corollary 2.2.4, the function

$$
\sum_{n=-\infty}^{\infty} \psi(n) \frac{e\left(n^{2} x\right)}{n^{\beta}}=\sum_{n \equiv \pm 1(12)} \frac{e\left(n^{2} x\right)}{n^{\beta}}-\sum_{n \equiv \pm 5(12)} \frac{e\left(n^{2} x\right)}{n^{\beta}}, \quad 3 / 2<\beta<5 / 2,
$$

and its real and imaginary parts are differentiable in $\mathbf{Q}$ and not differentiable in $\mathbf{R}-\mathbf{Q}$.

If $\psi=(\dot{\overline{5}})$, then, as $\psi$ is even and $|\psi|=5$,

$$
\Theta(z, 1, \psi)=\sum_{n=-\infty}^{\infty} \psi(n) e\left(n^{2} z\right) \in \mathcal{M}_{1 / 2}\left(\Gamma_{0}(100), \psi\right)-S_{1 / 2}\left(\Gamma_{0}(100), \psi\right) .
$$

In fact it can be checked that it is cuspidal exactly at the non-equivalent cusps 0 , $1 / 2,1 / 4,1 / 10,7 / 10,9 / 10,1 / 25,1 / 50$ and $1 / 100$. By Theorem 2.2, the function

$$
\sum_{n \equiv \pm 1(5)} \frac{e\left(n^{2} x\right)}{n^{\beta}}-\sum_{n \equiv \pm 2(5)} \frac{e\left(n^{2} x\right)}{n^{\beta}}, \quad 3 / 2<\beta<5 / 2,
$$

and its real and imaginary parts are differentiable at $x_{0}$ if and only if $x_{0}$ belongs to the $\Gamma_{0}(100)$-orbit of these cusps, which can be expressed in terms of congruence conditions as in Riemann's example.

We finish by giving the proofs of the results of this section.

Proof of Proposition 5.1. Note first that, by considering the Galois automorphism that sends $e(1 / b)$ into $e(a / b)$, for $\operatorname{gcd}(a, b)=1$ we have

$$
S(1, b)=0 \Leftrightarrow S(a, b)=0 .
$$

Hence $S(1, b) \neq 0$ implies $S(a, b) \neq 0$, and $\Theta$ is not cuspidal at $x_{0}=a / b$. For $k=1$, partial summation (use the periodicity of $S(\cdot, b)$ ) proves that the series formally defining $F^{\prime}\left(x_{0}\right)$ is not even Abel summable for $\alpha<3 / 2$; hence, by III.7.6 in [17], $F$ is not differentiable at $x_{0}$ (cf. [3]). This is also the case for $k>1$ when $\alpha<k / 2+1$, because the expansion at the cusp $x_{0}$ implies that the gamma integral representation

$$
F^{\prime}\left(x_{0}+i t\right)=\frac{(2 \pi)^{\alpha} i}{\Gamma(\alpha-1)} \int_{0}^{\infty} t^{\alpha-2}\left(\Theta\left(x_{0}+i(t+y)\right)-1\right) d t
$$

diverges as $y \rightarrow 0^{+}$(compare with the proof of Proposition 3.1).

Finally, if $S(1, b)=0$, then $\Theta$ is cuspidal at $x_{0}=a / b$ and, by the formula following Theorem $2.2, F$ is differentiable at $x_{0}$ in the indicated range.

Proof of Proposition 5.2. An elementary computation proves that for $\Re s>1 / 2$

$$
-\frac{1}{1^{2 s}}+\frac{1}{2^{2 s}}-\frac{1}{3^{2 s}}+\frac{1}{4^{2 s}}-\ldots=\left(2^{1-2 s}-1\right) \zeta(2 s),
$$

and by the gamma integral

$$
\frac{(-1)^{n}}{n^{\beta-2}}=\frac{(2 \pi)^{\beta / 2-1}}{i \Gamma(\beta / 2)} \int_{0}^{\infty} t^{\beta / 2-1} 2 \pi i n^{2} e\left(n^{2}(1 / 2+i t)\right) d t .
$$

Hence

$$
\frac{(2 \pi)^{\beta / 2}}{\Gamma(\beta / 2)} \int_{0}^{\infty} t^{\beta / 2-1} \Theta^{\prime}(1 / 2+i t) d t=2 \pi i\left(2^{3-\beta}-1\right) \zeta(\beta-2)
$$

and, according to Theorem 2.2, the left hand side is $F^{\prime}(1 / 2)$. 
Proof of Proposition 5.3. With a change of variable $x \mapsto 2^{-s} x$ we can always assume $s=0$.

Writing $\vec{n}=b_{1} \vec{n}_{1}+b_{2} \vec{n}_{2}$ with $\vec{n}_{1} \bmod b_{2}$ and $\vec{n}_{2} \bmod b_{1}$, it is easy to check that

$$
S\left(1, b_{1} b_{2}\right)=S\left(b_{2}, b_{1}\right) S\left(b_{1}, b_{2}\right)
$$

for $\operatorname{gcd}\left(b_{1}, b_{2}\right)=1$.

As $Q$ is diagonalizable in $\mathbf{Z} / p^{m} \mathbf{Z}$ for $p$ an odd prime (by completing squares), the explicit evaluation of Gauss sums can be applied to get

$$
S\left(1, p^{m}\right) \neq 0 .
$$

Hence, by (5.1), the above multiplicative property, Theorem 2.2 and Corollary 2.2.4, $\Re F$ and $\Im F$ are differentiable at $x_{0}$ if and only if for the highest power of 2 dividing the denominator of $x_{0}$, say $2^{t}$, we have

$$
S\left(1,2^{t}\right)=0 .
$$

Therefore it is enough to prove that this relation does not hold if $r=0$ and, for $r \neq 0$, it is fulfilled if and only if $t=1$ or $t=r-1$.

If $r \neq 0$, then $B$ is even, $4 \mid D$ and (perhaps exchanging $A$ and $C$ ) we can assume that $A$ is odd. Hence $Q$ can be diagonalized in $\mathbf{Z} / 2^{t} \mathbf{Z}$ (again by completing squares) as $A x^{2}+A^{\prime}(D / 4) y^{2}$ with $A$ and $A^{\prime}$ odd. So, by the multiplicative property and (5.1),

$$
S\left(1,2^{t}\right)=0 \Leftrightarrow \sum_{n \bmod 2^{t}} e\left(\frac{n^{2}}{2^{t}}\right) \cdot \sum_{m \bmod 2^{t+2}} e\left(\frac{D m^{2}}{2^{t+2}}\right)=0,
$$

which, by the explicit evaluation of Gauss sums, holds only for $t=1$ or $t=r-1$ (note that $r>1$ ).

It remains only to prove that $S\left(1,2^{t}\right) \neq 0$ if $r=0$. We proceed by induction on $t$. For $t=0$ or $t=1$, the result is trivial (note that $B$ is odd). If $t>1$, writing $\vec{n}=2^{t-1} \vec{a}+\vec{b}$ with $\vec{a}=\left(a_{1}, a_{2}\right), \vec{b}=\left(b_{1}, b_{2}\right)$, we have

$$
S\left(1,2^{t}\right)=\sum_{\vec{b} \bmod 2^{t-1}} \sum_{\vec{a} \bmod 2} e\left(\frac{B\left(a_{1} b_{2}+a_{2} b_{1}\right)}{2}\right) e\left(\frac{Q(\vec{b})}{2^{t}}\right) .
$$

As the innermost sum vanishes if $b_{1}$ and $b_{2}$ are not simultaneously even, we conclude that $S\left(1,2^{t}\right)=2 S\left(1,2^{t-2}\right)$.

\section{§6. SOME GRAPHICS}

In this section we present several pictures of some of the foregoing functions. Regarding the computational aspects, the Fourier coefficients of the automorphic forms corresponding to elliptic curves were obtained by use of the PARI package (the author thanks E. González Jiménez for his invaluable help), and partial sums have been calculated with simple FORTRAN programs, giving data output that was finally plotted using the MATLAB package.

First of all, we consider one of the "simplest" elliptic curves: one with minimal conductor $N=11$, given by the equation

$$
y^{2}+y=x^{3}-x^{2}-10 x-20 .
$$




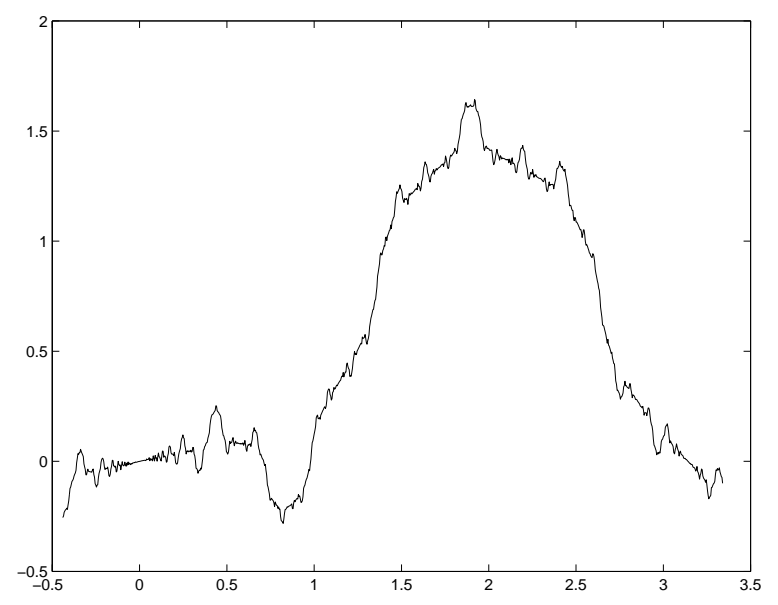

FiguRE 1.

Let $a_{n}$ be the coefficients of its Hasse-Weil $L$-function, and let

$$
g(x)=\sum_{n=1}^{\infty} \frac{a_{n}}{n^{7 / 4}} \sin (n x)
$$

According to Proposition 2.4, $g$ is differentiable exactly at rational multiples of $\pi$, and its graph has dimension 5/4. Plotting $g$, we obtain Figure 1.

We can see the differential behavior at $x=0$ as a quick oscillation with shrinking amplitude around the origin. The same phenomenon can be observed or guessed in this picture, in a self-similar way, around $2 \pi / 11,4 \pi / 11,2 \pi / 4,6 \pi / 11,2 \pi / 3$, $8 \pi / 11,10 \pi / 11$ and $\pi$. With the help of a computer we can check that by enlarging the drawing (typically with a very big scale) the same kind of oscillatory behavior appears around these and other rational multiples of $\pi$. For instance, the enlarged graphs around $x=\pi$ and $x=8 \pi / 11$ are shown in Figure 2 (note the different scale).

A natural question is, Why can we observe the oscillation showing differentiability to the naked eye only at the aforementioned points on the original whole graph of $g$ ? An answer can be provided by examining the use of Lemma 3.3 in the proof of Theorem 2.2: note that if $z$ tends (non-tangentially) to a cusp $a / b$ then, according to Lemma 3.3, a cusp form $f(z)$ goes to zero more quickly when the denominator $b$ is smaller and when the width of the cusp, $\sqrt{K}$, is larger. In our case, the underlying group is $\Gamma=\Gamma_{0}(11)$, and there are only two cusps up to equivalence by $\Gamma$, one of width 1 and one of width 11 . The cusps $x=0,1 / 4,1 / 3,1 / 2$ are equivalent to the first one, and $x=1 / 11,2 / 11,3 / 11,4 / 11,5 / 11$ are equivalent to the second. Hence the magnifying factor needed to observe the graph of $g(2 \pi x)$ around these latter points is, roughly speaking, one eleventh the size which should correspond to the size of their denominators.

Under the (weak) Birch-Swinnerton-Dyer conjecture (see [9]), using Corollary 2.2.2, we have that an elliptic curve over $\mathbf{Q}$ contains infinitely many rational points if and only if $B_{2}$ (defined as in Proposition 2.4) is flat around the origin, i.e., $B_{2}^{\prime}(0)=0$. This allows a geometrical representation of the conjecture. For instance, 

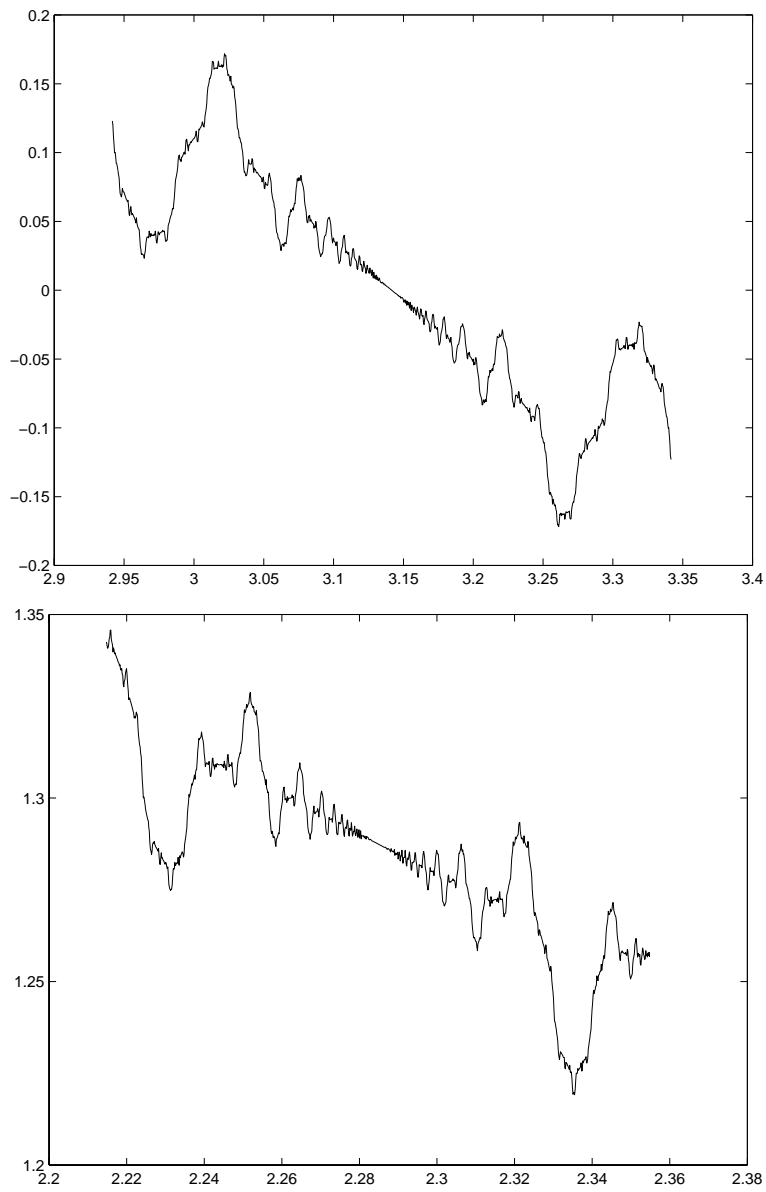

FiguRE 2.

consider the elliptic curves

$$
E_{1}: y^{2}+y=x^{3}+4 x-6, \quad E_{2}: y^{2}+y=x^{3}-x .
$$

The graphs of $B_{2}$ in a neighborhood of the origin are shown respectively in Figure 3 , which reflects the fact that $E_{1}$ contains only finitely many rational points while $E_{2}$ contains infinitely many. The scale factor to see this phenomenon depends on the size of the conductor.

The theta function case produces examples that are easily generated with a computer. One of the simplest is Riemann's example, shown in Figure 4. The graph illustrates clearly the differentiability of the function at $x=1 / 2$ and its absence at $x=0$.

One can study the nature of the oscillatory behavior around points of differentiability. Heuristically, if the cusp $\mathfrak{a}=0$ is a differentiability point of $f_{\alpha}$, the expansion of $f$ at this cusp suggests an oscillation with varying frequencies of the type $e(C / x)$ (note that $\sigma_{\mathfrak{a}} z=-C / z$ ), and the formula after Theorem 2.2 suggests that, in suitable ranges, the amplitude has the order $|x|^{2 \alpha-r}$. Hence an approximation like $f_{\alpha}(0)+f_{\alpha}^{\prime}(0) x+C^{\prime}|x|^{2 \alpha-r} e(C / x)$ could be expected for $x$ small enough, 

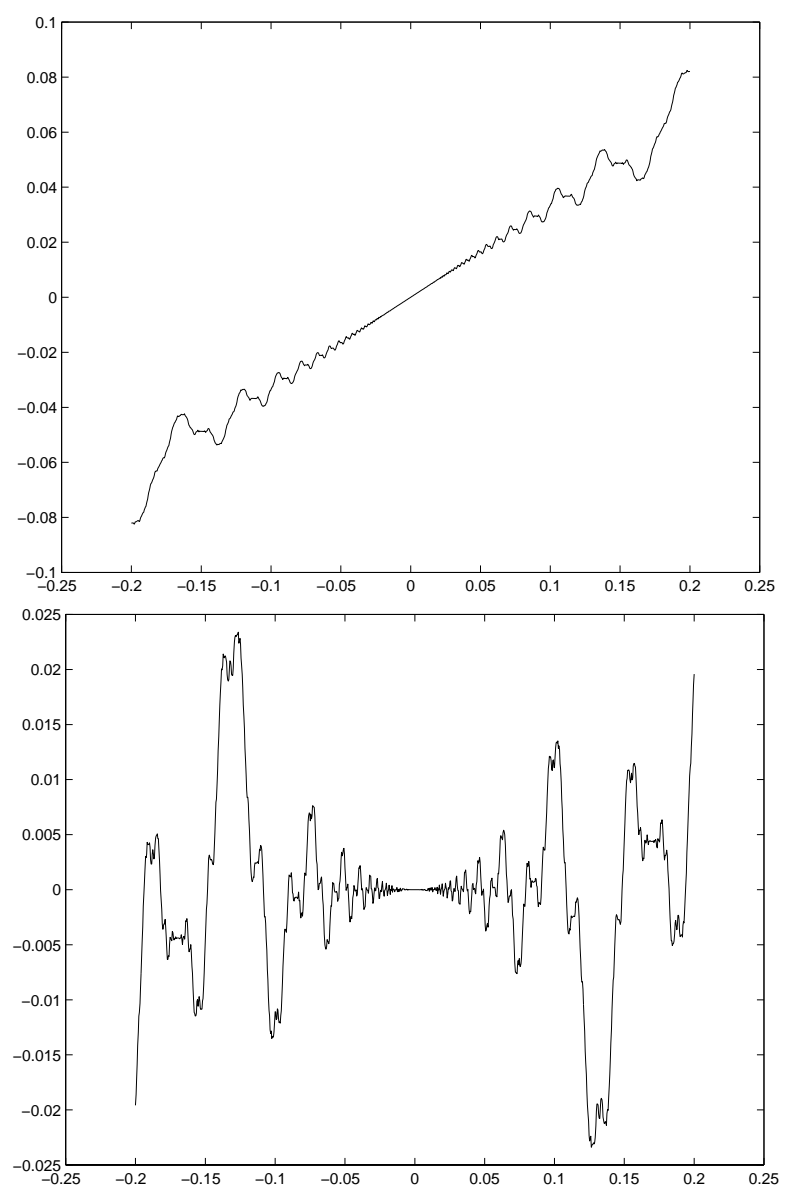

Figure 3.

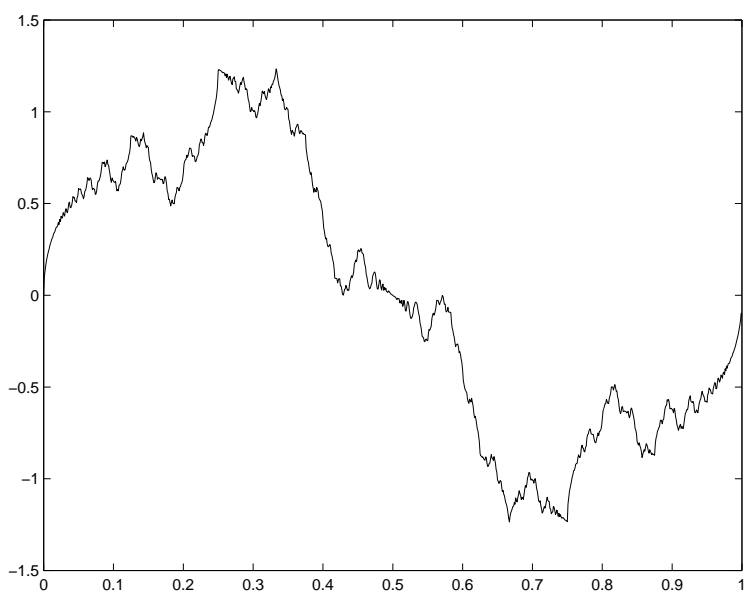

FiguRE 4. 

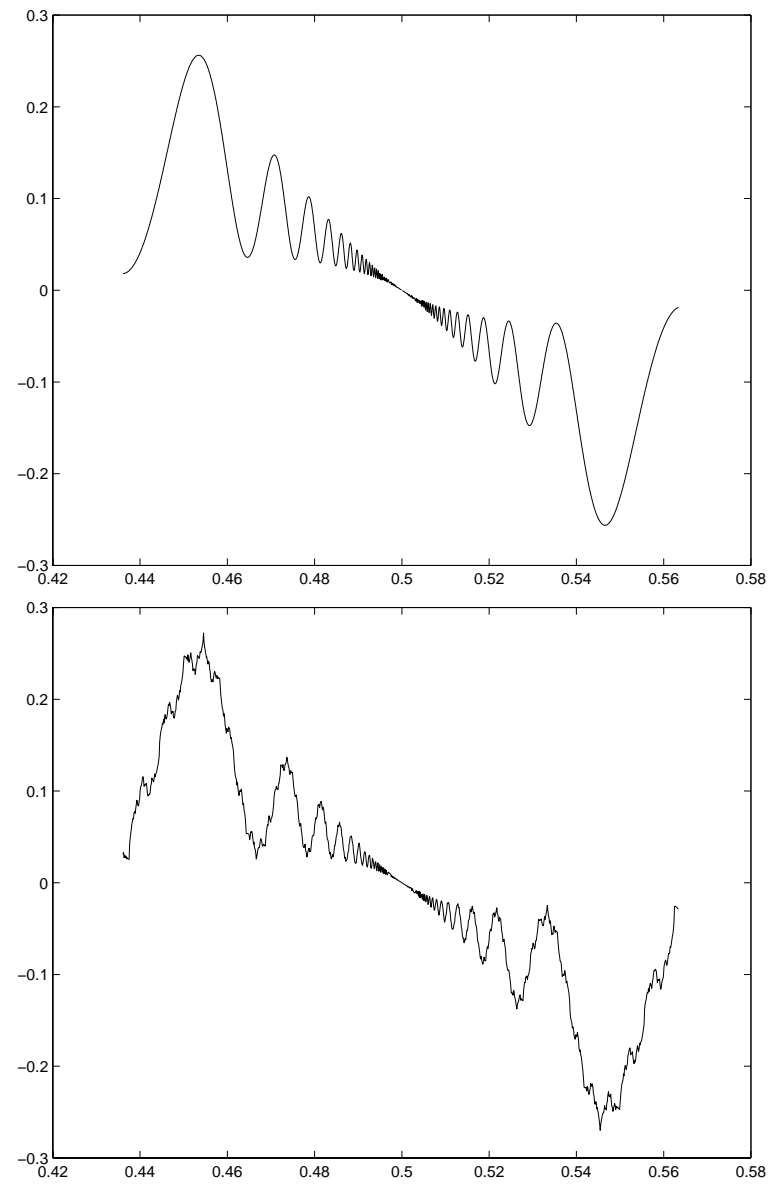

FIGURE 5 .

and similar expansions on small neighborhoods of other differentiability points. For instance, for Riemann's example $\alpha=1, r=1 / 2, f_{\alpha}(1 / 2)=0$ and $f_{\alpha}^{\prime}(1 / 2)=-\pi$. Figure 5 shows the graph of $g(x)=-\pi(x-1 / 2)+4|2 x-1|^{3 / 2} \sin (2 x-1)^{-1}$ and the actual graph of the Riemann's example around $x=1 / 2$. It is clear that they have the same behavior.

\section{ACKNOWLEDGEMENTS}

The author is deeply indebted to E. Valenti for helpful encouragement, and to H. Diamond for his kind help in preparing the last version of this work.

\section{REFERENCES}

1. H. Brézis, Analyse fonctionnelle: théorie et applications, Masson, Paris, 1983. MR 85a:46001

2. P. I. Butzer and E. I. Stark, "Riemann's example" of a continuous nondifferentiable function in the light of two letters (1865) of Christoffel to Prym, Bull. Soc. Math. Belg. 38 (1986), 45-73. MR 88d:01007

3. F. Chamizo and A. Córdoba, Differentiablity and dimension of some fractal Fourier series, Adv. in Math. 142 (1999), 335-354. MR 2000d:42002 
4. K. J. Falconer, The geometry of fractal sets, Cambridge Tracts in Mathematics, vol. 85, Cambridge University Press, 1986. MR 88d:28001

5. J. R. Ford, Fractions, Amer. Math. Monthly 45 (1938), 586-601.

6. J. Gerver, The differentiability of the Riemann function at certain rational multiples of $\pi$, Amer. J. Math. 92 (1970), 33-55. MR 42:434

7. G. H. Hardy, Weierstrass's non-differentiable functions, Trans. Amer. Math. Soc. 17 (1916), 301-325.

8. G. H. Hardy and E. M. Wright, An introduction to the theory of numbers, fifth edition, Oxford University Press, 1979. MR 81i:10002

9. D. Husemöller, Elliptic curves, Graduate Texts in Mathematics, vol. 111, Springer-Verlag, 1987. MR 88h:11039

10. H. Iwaniec, Topics in classical automorphic forms, Graduate Studies in Mathematics, vol. 17, American Mathematical Society, Providence, RI, 1997. MR 98e:11051

11. W. C. W. Li, Number theory with applications, Series on University Mathematics, vol. 7, World Scientific, Singapore, 1996. MR 98b:11001

12. M. V. Melián and D. Pestana, Geodesic excursions into cusps in finite-volume hyperbolic manifolds, Michigan Math. J. 40 (1993), 77-93. MR 94d:53067

13. W. Rudin, Real and complex analysis, third edition, McGraw-Hill, New York, 1987. MR 35:1420 (1st ed.); MR 88k:00002

14. J.-P. Serre and H. Stark, Modular forms of weight $1 / 2$, Lecture Notes in Mathematics 627, Springer-Verlag, 1977, 29-68. MR 57:12400

15. G. Shimura, Introduction to the arithmetic theory of automorphic functions, Princeton University Press, 1971. MR 47:3318

16. K. Weierstrass, Über continuirliche Functionen eines reellen Arguments, die für keinen Werth des Letzteren einen bestimmten Differentialquotienten besitzen (1872); English translation included in: Classics on Fractals (Ed., G.A. Edgar), Addison-Wesley Publishing Company, 1993.

17. A. Zygmund, Trigonometric series I, II, second edition, latest reprint, Cambridge University Press, 1990. MR 58:29731 etc.

Departamento de Matemáticas, Facultad de Ciencias, Ciudad Universitaria de CanTOBLANCO, MADRID 28049, SPAIN

E-mail address: fernando.chamizo@uam.es 